1 What are we missing by using hydrophilic enrichment? Improving bacterial glycoproteome coverage using total proteome and FAIMS analysis.

4 Ameera Raudah Ahmad Izaham ${ }^{1}$ Ching-Seng Ang ${ }^{2}$, Shuai Nie², Lauren E. Bird ${ }^{1}$, Nicholas A.

5 Williamson ${ }^{2}$ and Nichollas E. Scott ${ }^{1 \#}$

$7 \quad{ }^{1}$ Department of Microbiology and Immunology, University of Melbourne at the Peter Doherty

8 Institute for Infection and Immunity, Melbourne 3000, Australia

$9{ }^{2}$ Melbourne Mass Spectrometry and Proteomics Facility, The Bio21 Molecular Science and

10 Biotechnology Institute, University of Melbourne, Parkville, VIC, 3010, Australia.

20 Keywords: Glycoproteomics, FAIMS, HILIC, Glycopeptide enrichment, Bacterial glycosylation,

\title{
21 Burkholderia
}




\section{ABSTRACT}

24 Hydrophilic Interaction Liquid Chromatography (HILIC) glycopeptide enrichment is an

25 indispensable tool for the high-throughput characterisation of glycoproteomes. Despite its utility,

26 HILIC enrichment is associated with a number of short comings including requiring large amounts

27 of starting material, potentially introducing chemical artefacts such as formylation, and

28 biasing/under-sampling specific classes of glycopeptides. Here we investigate HILIC enrichment

29 independent approaches for the study of bacterial glycoproteomes. Using three Burkholderia

30 species (B. cenocepacia, B. dolosa and B. ubonensis) we demonstrate that short aliphatic $O$-linked

31 glycopeptides are typically absent from HILIC enrichments yet are readily identified in whole

32 proteome samples. Using Field Asymmetric Waveform IMS (FAIMS) fractionation we show that

33 at low compensation voltages (CVs) short aliphatic glycopeptides can be enriched from complex

34 samples providing an alternative means to identify glycopeptides recalcitrant to hydrophilic

35 based enrichment. Combining whole proteome and FAIMS analysis we show that the observable

36 glycoproteome of these Burkholderia species is at least $30 \%$ larger than initially thought.

37 Excitingly, the ability to enrich glycopeptides using FAIMS appears generally applicable, with the

$38 \quad \mathrm{~N}$-linked glycopeptides of Campylobacter fetus subsp. fetus also enrichable at low FAIMS CVs.

39 Taken together, these results demonstrate that FAIMS provides an alternative means to access

40 glycopeptides and is a valuable tool for glycoproteomic analysis. 


\section{INTRODUCTION}

46 Glycosylation is a ubiquitous protein modification found in all domains of life [1]. Over the last

47 two decades significant progress has been made in our understanding of glycosylation targets

48 leading to the establishment of the field of glycoproteomics [2, 3]. The growth of glycoproteomics

49 has been driven by the increasing sensitivity and speed of mass spectrometry (MS)

50 instrumentation as well as dramatic improvements in glycopeptide informatics [2-4]. These

51 advancements now enable intact glycopeptide analysis - the study of glycopeptides decorated

52 with their native [4], native-like $[5,6]$ or truncated $[7,8]$ glycoforms - and enables the qualitative

53 and quantitative assessment of glycosylation at a site-specific resolution. Intact glycopeptide

54 analysis has been critical for the study of glycosylation where the sites of modification are not

55 predictable based on amino acid sequence such as mucin O-linked glycosylation [7, 8], $\mathrm{O}$ -

mannosylation $[9,10]$ and $O$-glcNAcylation [11]. Although powerful, a key requirement for intact

57 glycopeptide analysis is the ability to enrich/isolate glycopeptides for downstream analysis.

59 To date, MS based characterisation of intact glycopeptides has been heavily dependent on glycan

60 centric glycopeptide enrichment methods [2-4, 12]. Enrichment has been proposed to be critical

61 for concentrating glycopeptides in even simple protein digests [13] to overcome the suppression

62 of glycopeptide ions by co-eluting non-glycosylated peptides [12]. A range of methods have been

63 developed to enrich glycopeptides from mixtures [14] including lectin-affinity based approaches

64 [15-17], reversible chemical coupling resins such as boronic acid based derivatives [18] and

65 irreversible chemical strategies such as hydrazine based chemistry followed by carbohydrate

66 selective hydrolysis [19]. One of the most widely used glycopeptide enrichment approaches is 
67 hydrophilic interaction liquid chromatography (HILIC) enrichment using zwitterionic hydrophilic

68 interaction liquid chromatography (ZIC-HILIC) resins [20]. Enrichment using ZIC-HILIC is the result

69 of hydrophilic partitioning of glycopeptides to an adsorbed "water layer" on the surface of the

70 ZIC-HILIC resin [21, 22]. For glycopeptides, hydrophilic partitioning is thought to be driven largely

71 by glycans making ZIC-HILIC enrichment compatible with a range of glycopeptides provided their

72 glycans are sufficiently hydrophilic [23]. This enrichment approach has been used for nearly two

73 decades [20, 24] for both eukaryotic [25-28] and prokaryotic [29-32] glycoproteomic studies.

74 Although ZIC-HILIC enrichment is considered by some to be unbiased for eukaryotic $N$-linked

75 glycoproteomics [12, 33], it has been previously shown to strongly favour large glycans [23] and

76 show poor retention of $O$-linked glycopeptides [34]. This bias has important ramifications

77 especially for the study of glycosylated substrates decorated with short glycans such as the

78 glycoproteomes of Burkholderia species [31, 35].

80 Protein glycosylation is increasingly recognised as a highly conserved feature of bacterial

81 physiology [36-39]. To date, studies of bacterial glycoproteomes have extensively used ZIC-HILIC

82 enrichment [29-32, 40-43] to characterise glycosylation substrates and glycan diversity. Using

83 ZIC-HILIC enrichment, we have previously shown over 100 proteins are glycosylated within

84 Burkholderia species [44]. Despite this insight, the complete repertoire of most Burkholderia

85 glycoproteomes are still unknown, hampering our understanding of the impacts observed when

86 glycosylation is disrupted $[31,45,46]$. Within members of the Burkholderia genus, two linear

87 trisaccharides composed of $\beta-G a l-(1,3)-\alpha-G a I N A c-(1,3)-\beta-G a I N A c$ and Suc- $\beta-G a l-(1,3)-\alpha-G a I N A c-$

88 (1,3)- $\beta$-GalNAc (where Suc is Succinyl) are utilized for protein glycosylation [31, 35]. The short 
89 length of these two glycans raises notable concerns about potential bias in the observable

90 glycoproteome within ZIC-HILIC enrichments [23, 34]. This concern, coupled with our recent

91 identification of extensive formylation artefacts as a result of ZIC-HILIC enrichment [44], suggests

92 alternative approaches - such as orthogonal fractionation - are required for the analysis of

93 bacterial glycopeptides.

94

95 Ion Mobility Spectrometry (IMS) is an increasingly utilised gas-phase fractionation approach

96 which enables the separation of analytes based on a combination of charge and collisional cross-

97 section [47]. Within proteomics, Field Asymmetric Waveform IMS (FAIMS) is one of the most

98 widely used IMS approaches [48] and has been used to increase proteome coverage [49-51],

99 reduce ratio compression within isobaric labelling experiments [52], improve the localisation of

100 PTMs $[53,54]$ as well as enrich modified peptides such as sumoylated [55] and cross-linked [56]

101 peptides. Recently a new FAIMS interface was introduced for Orbitrap instruments: the FAIMS

102 Pro interface [49-51]. This new implementation of FAIMS improves sensitivity and enables rapid

103 compensation voltage (CV) switching, making it ideal for proteomics studies and gas-phase

104 fractionation $[49,50]$. By applying multiple CVs in a single experiment, referred to from here in

105 as stepped FAIMS, this has been shown to increase protein identifications by up to $55 \%$ vs

106 without FAIMS implementation [49-51]. Previous iterations of FAIMS have been shown to enable

107 the separation of glycopeptide isomers $[57,58]$ as well as improve the mapping of bacterial

108 glycosylation sites within single proteins [59]. Due to the large collisional cross-section of

109 glycopeptides compared to unmodified peptides [60, 61], IMS has been suggested to provide a 
110 unique avenue for the sorting/enrichment of glycopeptides [60, 62]. However, to our knowledge

111 FAIMS has not been previously used for glycoproteomics studies.

113 In this work we explore ZIC-HILIC independent approaches for the characterisation of bacterial

114 glycoproteomes. By comparing the glycopeptides identified within three Burkholderia species (B.

115 cenocepacia, B. dolosa and B. ubonensis) with and without ZIC-HILIC enrichment, we find that

116 short aliphatic glycopeptides are under-represented within ZIC-HILIC enrichments. To improve

117 the identification of these glycopeptides we explore the use of FAIMS and demonstrate that at

118 low FAIMS CVs short/aliphatic glycopeptides can be readily enriched from total proteome

119 samples. By combining multiple FAIMS CVs within a single analytical method and the analysis of

120 total proteome samples, we demonstrate that the glycoproteome of these three Burkholderia

121 species are at least 30\% larger than previously thought. Similarly, glycopeptides decorated with

122 large glycans such as the $N$-linked hexasaccharides of Campylobacter fetus subsp. fetus are

123 equally enriched at low FAIMS CVs with the use of FAIMS providing comparable coverage of the

124 glycoproteome using only a fraction of the starting material. The ability to access complementary

125 glycoproteomes as well as enrich glycopeptides from low input amounts makes FAIMS a powerful

126 tool for glycoproteomic analysis. 


\section{EXPERIMENTAL PROCEDURES}

133 Bacterial strains and growth conditions: [63]. B. cenocepacia J2315; B. dolosa AU0158 and B.

134 ubonensis MSMB22 strains were grown overnight on LB agar at $37^{\circ} \mathrm{C}$ as previously described [31].

135 C. fetus subsp. fetus NCTC 10842 was grown on Brain-Heart Infusion medium (Hardy Diagnostics)

136 with 5\% defibrinated horse blood (Hemostat, Dixon, CA) under microaerobic conditions (10\%

$137 \mathrm{CO}_{2}, 5 \% \mathrm{O}_{2}, 85 \% \mathrm{~N}_{2}$ ) at $37^{\circ} \mathrm{C}$ as previously reported [64]. Details on the strains, their origins, 138 references and proteome databases are provided within Table 1.

Generation of bacterial lysates for glycoproteome analysis: Bacterial strains were grown to

141 confluency before flooding plates with $5 \mathrm{~mL}$ of pre-chilled sterile phosphate-buffered saline (PBS)

142 and bacterial cells collected by scraping. Cells were washed 3 times in PBS to remove media

143 contaminates, then collected by centrifugation at $10,000 \times g$ at $4^{\circ} \mathrm{C}$ for 10 min, and then snap

144 frozen. Snap frozen cells were resuspended in 4\% SDS, 100mM Tris pH 8.0, 20mM Dithiothreitol

145 (DTT) and boiled at $95^{\circ} \mathrm{C}$ with shaking at 2000rpm for $10 \mathrm{~min}$. Samples were clarified by

146 centrifugation at $17,000 \times g$ for $10 \mathrm{~min}$, the supernatants were then collected, and protein

147 concentrations determined by a bicinchoninic acid assay (Thermo Fisher Scientific, Waltham, MA,

148 USA). $1 \mathrm{mg}$ of protein from each sample was acetone precipitated by mixing one volume of

149 sample with 4 volumes of ice-cold acetone. Samples were precipitated overnight at $-20^{\circ} \mathrm{C}$ and

150 then spun down at $16,000 \times g$ for $10 \mathrm{~min}$ at $0^{\circ} \mathrm{C}$. The precipitated protein pellets were

151 resuspended in $80 \%$ ice-cold acetone and precipitated for an additional 4 hours at $-20^{\circ} \mathrm{C}$. Samples

152 were centrifuged at $17,000 \times g$ for $10 \mathrm{~min}$ at $0^{\circ} \mathrm{C}$, the supernatant discarded, and excess acetone 153 driven off at $65^{\circ} \mathrm{C}$ for $5 \mathrm{~min}$. 
Digestion of protein samples: Protein digestion was undertaken as previously described with minor alterations [29]. Briefly, dried protein pellets were resuspended in $6 \mathrm{M}$ urea, $2 \mathrm{M}$ thiourea in $40 \mathrm{mM} \mathrm{NH}_{4} \mathrm{HCO}_{3}$ then reduced for 1 hour with 20mM DTT, followed by alkylation with 40mM chloroacetamide for 1 hour in the dark. Samples were then digested with Lys-C (Wako Chemicals, Japan; $1 / 200 \mathrm{w} / \mathrm{w}$ ) for 3 hours before being diluted with 5 volumes of $40 \mathrm{mM} \mathrm{NH}_{4} \mathrm{HCO}_{3}$ and digested overnight with sequencing grade modified trypsin (Promega, Madison, WI, USA; 1/50 $\mathrm{w} / \mathrm{w})$. Digested samples were acidified to a final concentration of $0.5 \%$ formic acid and desalted

162 with $50 \mathrm{mg}$ tC18 Sep-Pak columns (Waters corporation, Milford, MA, USA) according to the 163 manufacturer's instructions. tC18 Sep-Pak columns were conditioned with 10 bed volumes of 164 Buffer B (0.1\% formic acid, 80\% acetonitrile), then equilibrated with 10 bed volumes of Buffer $A^{*}$ 165 (0.1\% TFA, $2 \%$ acetonitrile) before use. Samples were loaded on to equilibrated columns and then 166 columns washed with at least 10 bed volumes of Buffer $A^{*}$ before bound peptides were eluted

167 with Buffer B. Eluted peptides were aliquoted into samples for ZIC-HILIC enrichment or total 168 proteome analysis, then dried by vacuum centrifugation and stored at $-20^{\circ} \mathrm{C}$.

170 Reverse phase LC-MS/MS: Proteome samples were re-suspended in Buffer A* and separated 171 using a two-column chromatography set up composed of a PepMap100 C18 20 mm x $75 \mu \mathrm{m}$ trap 172 and a PepMap C18 500 mm x $75 \mu \mathrm{m}$ analytical column (Thermo Fisher Scientific). Samples were 173 concentrated onto the trap column at $5 \mu \mathrm{L} / \min$ for 5 minutes with Buffer $\mathrm{A}(0.1 \%$ formic acid, $2 \%$ 174 DMSO) and then infused into an Orbitrap Fusion ${ }^{\mathrm{TM}}$ Lumos $^{\mathrm{TM}}$ Tribrid $^{\mathrm{TM}}$ Mass Spectrometer (Thermo 
176 (Thermo Fisher Scientific). For stepped CV and whole proteome experiments, 185-minute

177 analytical runs were undertaken by altering the buffer composition from $2 \%$ Buffer B $(0.1 \%$ formic

178 acid, $77.9 \%$ acetonitrile, $2 \%$ DMSO) to $28 \%$ B over 150 minutes, then from $28 \%$ B to $40 \%$ B over

17910 minutes, then from $40 \%$ B to $100 \%$ B over 2 minutes. The composition was held at $100 \%$ B for

1803 minutes, and then dropped to $2 \%$ B over 5 minutes before being held at $2 \%$ B for another 15

181 minutes. These conditions are identical to the published ZIC-HILIC glycopeptide datasets [44] to

182 enable the direct comparison of glycopeptide coverage. For static FAIMS CVs and associated no

183 FAIMS control experiments, 125-minute analytical runs were undertaken by altering the buffer

184 composition from 2\% Buffer B (0.1\% formic acid, $77.9 \%$ acetonitrile, $2 \%$ DMSO) to $28 \%$ B over 90

185 minutes, then from $28 \%$ B to $40 \%$ B over 10 minutes, then from $40 \%$ B to $100 \%$ B over 2 minutes.

186 The composition was held at $100 \%$ B for 3 minutes, and then dropped to $2 \%$ B over 5 minutes

187 before being held at $2 \% \mathrm{~B}$ for another 15 minutes. For all experiments, the Lumos $^{\mathrm{TM}}$ Mass $^{\mathrm{s}}$

188 Spectrometer was operated in a data-dependent mode, automatically switching between the

189 acquisition of a Orbitrap MS scan (120,000 resolution) every 3 seconds and Orbitrap MS/MS HCD

190 scans of precursors (NCE 30\%, maximal injection time of $60 \mathrm{~ms}$, with an AGC of $200 \%$ and a

191 resolution of 15,000$)$. Glycan fragment ions $(204.087 ; 366.1396$ and $138.0545 \mathrm{~m} / \mathrm{z})$ product-

192 dependent MS/MS analysis [65] was used to trigger three additional scans of potential

193 glycopeptides; a Orbitrap EThcD scan (NCE 15\%, maximal injection time of 250 ms with an AGC

194 of $500 \%$ and a resolution of 30,000 using the extended mass range setting to improve the

195 detection of high mass glycopeptide fragment ions [61]); an ion trap CID scan (NCE 35\%, maximal

196 injection time of 40 ms with an AGC of 200\%) and a stepped collision energy HCD scan (using NCE

$19730 \%, 35 \%, 45 \%$ with a maximal injection time of 250 ms with an AGC of $500 \%$ and a resolution of 
30,000). For FAIMS experiments, static FAIMS analysis was undertaken using CVs of -20; -30; -40;

$-50 ;-60 ;-70 ;-80$ and -90 while stepped FAIMS experiments were undertaken switching between the CVs $-25 ;-35$ and -45 . All experiments were collected in triplicate.

Data Analysis - Glycopeptide identification: Raw data files were batch processed using Byonic

GB of RAM using a maximum of 16 cores for a given search. For all searches, a semi-tryptic N-

ragged specificity was set, and a maximum of two missed cleavage events allowed.

207 Carbamidomethyl was set as a fixed modification of cystine while oxidation of methionine was

208 included as a variable modification. A maximum mass precursor tolerance of 5 ppm was allowed

209 while a mass tolerance of up to $10 \mathrm{ppm}$ was set for HCD fragments and 20 ppm for EThcD

210 fragments. Burkholderia species searches were conducted allowing two O-linked glycans: Hex-

211 HexNAc-HexNAc (elemental composition: $\mathrm{C}_{22} \mathrm{O}_{15} \mathrm{H}_{36} \mathrm{~N}_{2}$, mass: 568.2115) and Suc-Hex-HexNAc-

212 HexNAc (elemental composition: $\mathrm{C}_{26} \mathrm{O}_{18} \mathrm{H}_{40} \mathrm{~N}_{2}$, mass: 668.2276), while $C$. fetus fetus searches

213 were conducted allowing two $N$-linked glycans: Hex-HexNAc4-diNAcBac (elemental composition:

$214 \mathrm{C}_{48} \mathrm{O}_{29} \mathrm{H}_{78} \mathrm{~N}_{6}$, mass: 1202.4813) and $\mathrm{HexNAC}_{5}$-diNAcBac (elemental composition: $\mathrm{C}_{50} \mathrm{O}_{29} \mathrm{H}_{81} \mathrm{~N}_{7}$,

215 mass: 1243.5078) where diNAcBac is the bacterial specific sugar 2,4-diacetamido-2,4,6

216 trideoxyglucopyranose [64]. To ensure high data quality, technical replicates were combined

217 using R (https://www.r-project.org/) and only glycopeptides with a Byonic score >300 were used

218 for further analysis. This cut-off is in line with previous reports highlighting that score thresholds

219 greater than at least 150 are required for robust glycopeptide assignments with Byonic [28, 67]. 
220 It should be noted that a score threshold of above 300 resulted in false discovery rates of less

221 than $1 \%$ for all combined datasets.

223 Data Analysis - iBAQ analysis: To assess the relative abundance of proteins, iBAQ analysis was

224 undertaken. Unenriched proteome datasets were processed using MaxQuant v1.6.3.4 [68]

225 searching against the reference $B$. cenocepacia strain J2315. Carbamidomethylation of cysteine

226 was set as a fixed modification while oxidation of methionine and acetylation of protein $\mathrm{N}$-termini

227 were allowed as variable modifications. An enzyme specificity of "trypsin/P" was set with a

228 maximum of 2 missed cleavage events. The resulting outputs were processed in the Perseus

229 v1.5.0.9 [69] analysis environment to remove reverse matches and common protein

230 contaminates prior to further analysis.

232 Peptide analysis, data visualization and data sharing: The previously published ZIC-HILIC

233 glycopeptide datasets [44] were used to compare the differences in glycopeptides observed with

234 and without ZIC-HILIC enrichment. It should be noted that the proteome digests within this work

235 are the same digests used for ZIC-HILIC enrichments in [44]. Analysis and visualization was

236 undertaken using R (https://www.r-project.org/) with the glycopeptide physicochemical

237 properties determined using the Peptides package [70] and data visualized using ggplot2 [71] and

238 ggpubr packages. The aliphatic index within the Peptides package is calculated by assessing the

239 relative volume occupied by aliphatic side chains within a peptide [72]. All mass spectrometry

240 proteomics data (Raw data files, Byonic search outputs and R Scripts) have been deposited into

241 the PRIDE ProteomeXchange Consortium repository $[73,74]$ with the dataset identifier: 
bioRxiv preprint doi: https://doi.org/10.1101/2020.07.22.216903; this version posted July 23, 2020. The copyright holder for this preprint (which

was not certified by peer review) is the author/funder, who has granted bioRxiv a license to display the preprint in perpetuity. It is made available under aCC-BY-ND 4.0 International license.

242 PXD020442 and are accessible with the reviewer account details: Username:

243 reviewer56784@ebi.ac.uk Password: aoATFQMQ.

244

245

246

247

248

249

250

251

252

253

254

255

256

257

258

259

260

261

262

263 


\section{RESULTS}

Discrete glycopeptide populations are observed within whole cell digests compared to ZIC-

HILIC enriched samples

268 Recently we noted glycopeptides could be readily identified without enrichment across a range

269 of bacterial proteomes [23, 78]. Given this, we sought to compare the glycopeptides observed

270 with and without enrichment for bacterial species focusing on members of the Burkholderia

271 genus such as $B$. cenocepacia J2315. Within whole cell digests of $B$. cenocepacia J2315 we find

272 that only $\sim 2 \%$ of all high-confidence PSMs (Byonic ${ }^{\mathrm{TM}}$ scores $^{2} 300$ ) correspond to glycopeptides

273 (Supplementary Figure 1A, Supplementary Table 1). Although a low proportion of all PSMs this

274 still corresponds to hundreds of individual glycopeptide PSMs identified within analytical runs

275 (Supplementary Figure 1B, Supplementary Table 1). Intuitively, we assumed that within these

276 whole cell digests of $B$. cenocepacia J2315, the observed glycopeptides would correspond to

277 previously identified glycopeptides within ZIC-HILIC enrichments, yet this was not the case.

278 Surprisingly, the majority of glycopeptides identified within whole cell digests corresponded to

279 novel glycoproteins/glycopeptides (Figure $1 \mathrm{~A}$ and $\mathrm{B}$ ), with manual inspection of previously

280 unreported glycopeptides supporting the correctness of these assignments (Supplementary

281 Figure $2 \mathrm{~A}-\mathrm{H}$ ). These results demonstrate that different subsets of the $B$. cenocepacia J2315

282 glycoproteome are observed with and without ZIC-HILIC enrichment.

284 To understand the differences in the glycopeptides identified with and without ZIC-HILIC 285 enrichment we first compared the relative abundances of glycoproteins using iBAQ values 286 derived from whole cell digests (Figure 1C, Supplementary Table 2). We reasoned that the 
287 dramatic difference in the glycopeptide profiles may be linked to glycoprotein abundances with

288 ZIC-HILIC enrichment potentially accessing glycopeptides from lower abundance glycoproteins.

289 Although the iBAQ values of glycoproteins observed within enriched samples are slightly lower

$290 \quad(P=0.0036 ;$ mean iBAQ value of glycoproteins identified in enriched samples $=28.045$ compared

291 to the mean $\mathrm{iBAQ}$ value of glycoproteins identified within unenriched samples $=28.918$,

292 Supplementary Table 3), the difference in the abundance is modest (Figure 1C), suggesting that

293 the difference in the $B$. cenocepacia glycopeptides profiles observed between samples is

294 independent of protein abundance. As previous reports have suggested that peptide sequences

295 can influence ZIC-HILIC glycopeptide enrichment $[23,75]$ we examined the physiochemical

296 properties of the peptide sequences revealing marked differences in the glycopeptides observed

297 between samples (Figure 1D). We found that glycopeptides observed within whole cell digests

298 are both more aliphatic and typically shorter in length, while the majority of ZIC-HILIC enriched

299 glycopeptides are both longer and less aliphatic $\left(P=5.10^{*} 10^{15}\right.$ and $6.68 * 10^{15}$ aliphaticity and

300 length respectively, Supplementary Table 4). Combined these results support that differences in

301 the physiochemical properties of glycopeptides influences their ability to be enriched by ZIC-HILIC

302 enrichment.

304 ZIC-HILIC incompatible glycopeptides are readily accessible using FAIMS fractionation

305 The findings that ZIC-HILIC enrichment provides access to only a subset of Burkholderia

306 glycopeptides suggests that alternative approaches are required for comprehensive analysis of

307 Burkholderia glycoproteomes. As glycopeptides were readily identified within whole cell digests

308 of B. cenocepacia, we reasoned that an orthogonal fractionation approach may identify 
309 previously overlooked glycopeptides. Since FAIMS enables gas-phase fractionation based on

310 charge/collision cross section [49, 50], we examined the charge state distributions of

311 glycopeptides identified from whole cell digests compared to ZIC-HILIC enrichments (Figure 2A

312 and B). We noted that glycopeptides observed within the whole cell digest were predominantly

313 of low charge states $(<+3)$. As previous work [50] has shown that +3 and +2 peptides are identified

314 across CVs of -20 to -120 with apexes at -80 and -45 respectively this suggested low FAIMS CVs

315 would enable enrichment of potential low charge state glycopeptides. To assess this, we assayed

316 the ability of B. cenocepacia glycopeptides to be identified under differing FAIMS CVs from -20

317 to -90 (Figure 2C, Supplementary Table 5 and 6). Consistent with our hypothesis we found that

318 FAIMS CVs of less then -50 readily enabled the isolation of glycopeptides (Figure 2C,

319 Supplementary Figure 3A). Interestingly, we also found that within individual analytical runs

320 (denoted with dots in Figure 2C) with FAIMS CVs of $-20,-30$ and -40 more unique glycopeptides

321 were identified per run than analysis without FAIMS. However, when the results of triplicate runs

322 were combined (denoted by the bars in Figure 2C), only the FAIMS CV -30 outperformed analysis

323 without FAIMS (Figure 2C). Similar to whole proteome digests, FAIMS-fractionated glycopeptides

324 typically corresponded to novel glycoproteins/glycopeptides that were not captured by ZIC-HILIC

325 enrichments (Figure 2D and E) and were again shorter and more aliphatic then ZIC-HILIC enriched

326 glycopeptides (Supplementary Figure 3B). Within FAIMS CVs we noted that, consistent with

327 previous reports [50], the overlap between $10 \mathrm{CV}$ fractions was modest with a large proportion

328 of glycopeptides unique to a single CV (Figure 3A and B). Taken together these results suggest

329 that low FAIMS CVs provide access to ZIC-HILIC inaccessible $B$. cenocepacia glycopeptides yet the

330 coverage of the glycoproteome within a single FAIMS CV is limited. 


\section{Using stepped FAIMS improves the glycoproteome coverage of Burkholderia species}

332 Since individual FAIMS CVs provided access to only a subset of $B$. cenocepacia glycopeptides, we

333 reasoned that using stepped FAIMS may improve glycopeptide identifications. Comparing the

334 performance of stepped FAIMS, unenriched and ZIC-HILIC enriched digests we find that stepped

335 FAIMS leads to a $>30 \%$ increase in unique glycopeptides compared to unenriched samples (Figure

336 4A, Supplementary Table 7). As with static FAIMS CVs this improvement was predominantly

337 within glycopeptides not previously observed within ZIC-HILIC enrichments with $>60 \%$ of 338 glycopeptides unique to stepped FAIMS (Figure 4B). Interestingly, we find that the glycopeptides

339 identified in both stepped FAIMS and unenriched samples provide access to unique glycopeptide

340 subsets compared to ZIC-HILIC enrichments (Figure 4C). Analysis of the physiochemical

341 properties of glycopeptides observed using each approach highlights that the glycopeptides

342 observed within B. cenocepacia with stepped FAIMS and unenriched samples are largely similar,

343 favouring shorter and more aliphatic glycopeptides compared to the larger and less aliphatic

344 glycopeptides observed with ZIC-HILIC enrichment (Figure 4D, Supplementary Table 8). The

345 modest overlap of unique glycopeptides observed with stepped FAIMS and analysis without

346 FAIMS fractionation illustrates that the glycoproteome of $B$. cenocepacia is larger than initially

347 thought $[31,44]$, and that ZIC-HILIC enrichment fails to capture a significant proportion of the 348 Burkholderia glycoproteome.

350 To further confirm this bias within ZIC-HILIC enrichments we assessed two additional

351 Burkholderia species recently assessed using ZIC-HILIC based enrichment: $B$. dolosa and $B$.

352 ubonensis [44]. Consistent with our observations within B. cenocepacia both stepped FAIMS and 
353 unenriched samples revealed multiple unique glycopeptides/glycoproteins absent within ZIC-

354 HILIC enrichments (Figure 5A to B; Supplementary Tables 9-13). As with B. cenocepacia, these

355 newly observed glycopeptides in each Burkholderia species are typically shorter and/or more

356 aliphatic than glycopeptides observed within ZIC-HILIC enrichments (Supplementary Figure 4).

357 These results demonstrate that at least $30 \%$ of the glycoproteome of Burkholderia species are

358 not observed within ZIC-HILIC enrichments, making orthogonal approaches such as stepped

359 FAIMS valuable tools to access uncharacterised regions of Burkholderia glycoproteomes.

\section{FAIMS fractionation improves the identification of bacterial $\mathbf{N}$-linked glycoproteomes}

362 Finally, as FAIMS improved the identification of glycopeptides within Burkholderia

363 glycoproteomes, we wondered if other bacterial glycoproteomes would also benefit from FAIMS

364 fractionation. To assess this we used C. fetus subsp. fetus NCTC 10842 which is known to N365 glycosylate proteins with two glycans composed of $\beta$-GlcNAc- $\alpha 1,3-[$ GIcNAc1,6-]GIcNAc- $\alpha 1,4-$

366 GalNAc- $\alpha 1,4-G a I N A c-\alpha 1,3-d i N A c B a c \quad$ and $\beta-G l c N A c-\alpha 1,3-[G \mid c 1,6-]$ GlcNAc- $\alpha 1,4-G a I N A c-\alpha 1,4-$

367 GalNAc- $\alpha 1,4-d i N A c B a c$ [64]. As with the trisaccharide decorated glycopeptides of Burkholderia,

368 we noted a marked enrichment of C. fetus subsp. fetus glycopeptides within low CVs FAIMS

369 fractions compared to matching analytical runs without FAIMS (Figure 6A, Supplementary Table

37014 and 15). As observed within Burkholderia glycoproteomes, the use of stepped FAIMS

371 improved the detection of unique glycopeptides compared to unenriched samples with matching

372 analytical gradients (Figure 6B, Supplementary Table 16). Excitingly, we find that stepped FAIMS

373 enabled the identification of a large proportion of previously identified glycoproteins and

374 glycopeptides from ZIC-HILIC enrichments (Figure 6C, Supplementary Table 17) while requiring 
375 significantly less material $(2 \mu \mathrm{g}$ per stepped FAIMS run compared to $300 \mu \mathrm{g}$ per ZIC-HILIC

376 enrichment). These results demonstrate that large glycan-containing glycopeptides are enriched

377 at low FAIMS CVs in a similar manner to glycopeptides decorated with shorter glycans, and this

378 supports the general utilisation of FAIMS fractionation for isolating bacterial glycopeptides.

\section{Discussion}

381 Over the last decade, glycopeptide-based enrichment approaches have increasingly been used

382 to track and characterise microbial glycosylation events [42, 44, 64, 76]. Although enrichment

383 strategies enable high-throughput analysis of glycosylation, we demonstrate here that

384 hydrophilic enrichment provides only partial access to some microbial glycoproteomes. In light

385 of these findings we evaluated the application of FAIMS fractionation using the FAIMS Pro

386 interface [49-51] as an orthogonal approach for probing bacterial glycosylation. We find that for

387 bacterial $\mathrm{N}$ - and $\mathrm{O}$-linked glycans, low FAIMS CVs enables the enrichment of glycopeptides from

388 whole proteome samples. By utilising a stepped FAIMS approach in concert with direct analysis

389 of whole cell digests we show that the glycoproteomes of three Burkholderia species are $>30 \%$

390 larger than initially thought and that the majority of the known glycoproteome of $C$. fetus subsp.

391 fetus can be directly monitored from whole proteome digests.

393 To date, multiple studies have shown that glycosylation events can be readily detected within

394 proteome samples using variable modification searches $[77,78]$ or open searching approaches

395 [79-81]. These previous findings and our work here highlight that a wealth of glycoproteome 396 information can be gained from unenriched samples, despite the known issue of glycopeptide 
397 ion suppression [12]. Experimentally, it has been shown that glycopeptide signals can be

398 suppressed up to ten-fold compared to unmodified forms of the identical peptide [82] yet this

399 issue is addressable. As current state of the art MS instruments enable the identification of

400 potential glycopeptides by the presence of known sugar oxonium ions [65] this allows

401 glycopeptide spectra to be re-collected with altered settings generating high-quality MS/MS data

402 for even low abundance signals. Since FAIMS fractionation occurs post ionisation, it should be

403 noted that ion suppression is not prevented but is likely being mitigated by the improvement in

404 the signal to noise of analytes. As the amount of ion suppression is impacted by the complexity

405 of the attached glycans of glycopeptides [82], it is reasonable to assume that some

406 glycoproteomes will be more amenable to FAIMS analysis then others. Our work here

407 demonstrates that glycopeptides of Burkholderia species and C. fetus subsp. fetus appear readily

408 amenable to FAIMS enrichment, although further work will be required to establish general

409 trends for specific classes of glycosylation.

411 The ability to enrich glycopeptides independent of off-line glycan-centric enrichment strategies

412 opens up exciting possibilities for the study of previously recalcitrant glycoproteomes. Previous

413 comparative genomic [83] as well as ex vivo studies [84] have identified a number of potential

414 bacterial glycosylation systems for which the native substrates and glycans have yet to be

415 defined. For example, in Vibrio cholerae a functional $O$-linked glycosylation system has been

416 identified [84] and shown to be required for cholera toxin secretion [85], yet the glycoproteins

417 of this system are still unknown. Due to the diversity of glycans used for bacterial protein 418 glycosylation $[29,30,35,41,42,59,64,76,86,87]$ it is not surprising that a single approach such 
419 as ZIC-HILIC enrichment is inapplicable to all glycosylation systems or substrates within a single

420 glycoproteome. Indeed, within this study we note that the bias within Burkholderia ZIC-HILIC

421 enrichments does not appear to occur within ZIC-HILIC enrichments of C. fetus subsp. fetus

422 (Supplementary Figure 5, Supplementary Table 17). This difference is likely due to the larger more

423 hydrophilic glycans of $C$. fetus subsp. fetus which are retained more effectively during ZIC-HILIC

424 enrichment. Thus, the diversity within microbial glycosylation systems means that multiple

425 approaches are likely needed, with FAIMS providing an orthogonal approach for undertaking high

426 throughput microbial glycoproteomic analysis.

428 The observation that FAIMS enables the enrichment of glycopeptides at a proteome scale has

429 not explicitly been shown before although multiple IMS studies have highlighted this potential.

430 In 2013 the Hill group demonstrated the ability of IMS to separate glycosylated and non-

431 glycosylated components based on differences in collisional cross section [62], noting that this

432 could provide a novel strategy for high throughput glycopeptide identification. This concept has

433 been echoed in more recent studies which have shown that the ion mobility of low charge state

434 glycopeptides can be dramatically impacted by the presence of glycans [60]. Our finding that

435 FAIMS enables access to novel glycopeptides is consistent with previous studies of single

436 glycoproteins [59] and we demonstrate that this can be extended to the analysis of

437 glycoproteomes. A clear benefit of the recent FAIMS Pro interface is the rapid CV switching

438 enabling multiple CVs to be accessible within a single analytical framework. We find stepped

439 FAIMS approaches improves the detection of unique glycopeptides (Figure $4 \mathrm{~A}$ and $6 \mathrm{~A}$ ) yet it

440 should be noted that the optimal stepping regimes for glycopeptide identification is still unclear. 
441 It is plausible that further improvements in glycoproteome coverage may be possible by

442 optimising the CV switching to ensure minimal overlap between steps, while still focusing on the

443 low CV region where glycopeptides are enriched. Our results provide support for the notion that

444 FAIMS can improve our ability to study previously overlooked regions of glycoproteomes,

445 warranting further investigation of these methods for the field of glycoproteomics. 


\section{Figures}

Figure 1: Discrete glycopeptides are observed within unenriched and ZIC-HILIC enriched bacterial samples. A and B) The overlap of glycoproteins (A) and glycopeptides (B) identified without and with ZIC-HILIC enrichment demonstrates the majority of glycopeptides/glycoproteins observed within unenriched samples are unique to those observed within ZIC-HILIC enrichments. C) iBAQ measurements demonstrate only modest differences in the relative abundance of glycoproteins observed with and without ZIC-HILIC enrichment. The mean iBAQ values for each group are denoted by the dotted lines. D) The physiochemical properties of glycopeptides observed with and without ZIC-HILIC enrichment demonstrates glycopeptides observed without enrichment are shorter and more aliphatic then glycopeptides observed with ZIC-HILIC enrichment.

Figure 2. FAIMS fractionation enables the enrichment of glycopeptides within B. cenocepacia J2315. A and B) Charge state distributions of glycopeptides observed without ZIC-HILIC enrichment (A) and glycopeptides observed with ZIC-HILIC enrichment (B). C) $2 \mathrm{hr}$ analytical gradients of FAIMS fractionation at different CVs demonstrate glycopeptides are enriched at low FAIMS CVs compared to $2 \mathrm{hr}$ analytical gradients of analysis without FAIMS fractionation. Red dots denote the numbers of unique glycopeptides in individual replicates at each FAIMS CV. Blue dots denote the numbers of unique glycopeptides in individual replicates from analysis without FAIMS. Bars represent the total number of unique glycopeptides identified under each condition. D and E) FAIMS fractionated samples enable the identification of glycoproteins (D) and glycopeptides (E) not observed within ZIC-HILIC enrichments.

Figure 3. FAIMS CV fractions provide access to unique glycopeptide pools. A) Analysis of static CVs observed to contain glycopeptides demonstrates that a large proportion of glycopeptides are unique to a single $\mathrm{CV}$ fraction. B) Pie chart of unique glycopeptides observed within static FAIMS Comparison of $3 \mathrm{hr}$ analytical gradients of ZIC-HILIC enrichments, stepped FAIMS and unenriched digests. Stepped FAIMS improves the identification of unique glycopeptides compared to unenriched samples. Individual replicates are denoted by dots and the combined number of unique glycopeptides within an approach are denoted by bars B) Comparison of the overlap in glycoproteins and glycopeptides identified with stepped FAIMS compared to ZIC-HILIC enrichments reveals stepped FAIMS enables access to glycopeptides/glycoproteins not observed within ZIC-HILIC enrichments. C) Overlap of glycopeptides observed within ZIC-HILIC enrichments, stepped FAIMS and analysis without FAIMS fractionation identifies that unique pools of glycopeptides are identified within each approach. D) The physiochemical properties of glycopeptides demonstrate that stepped FAIMS and analysis of unenriched digests allows the identification of typically more aliphatic and shorter glycopeptides. 
Figure 5. Comparison of glycoproteome coverage of Burkholderia species with ZIC-HILIC enrichment, Stepped FAIMS and analysis of unenriched samples. Analysis of B. dolosa and $B$. ubonensis demonstrate that multiple novel glycopeptides/glycoproteins are readily identified using stepped FAIMS and unenriched whole proteome samples.

Figure 6. FAIMS fractionation of $\boldsymbol{C}$. fetus subsp. fetus $\boldsymbol{N}$-linked glycopeptides. A) Static FAIMS CVs demonstrate glycopeptides are enriched at low FAIMS CVs compared to analysis without FAIMS fractionation within $2 \mathrm{hr}$ analytical runs. Red dots denote the numbers of unique glycopeptides in individual replicates in each FAIMS CV. Blue dots denote the numbers of unique glycopeptides in individual replicates from analysis without FAIMS. Bars represent the total number of unique glycopeptides identified under each condition. B) Comparison of $3 \mathrm{hr}$ analytical runs of ZIC-HILIC enrichment, stepped FAIMS and analysis of unenriched whole proteome samples demonstrates an improvement in unique glycopeptides identified with stepped FAIMS compared to analysis without FAIMS fractionation. C) Comparison of the overlap in glycoproteins and glycopeptides identified with stepped FAIMS compared to ZIC-HILIC enrichments. At both the glycopeptide and glycoprotein level, stepped FAIMS enables identification of $>60 \%$ of the known glycoproteome.

Table 1. Strain list

\begin{tabular}{|c|c|c|c|}
\hline Strains & Source & Reference & Proteome database \\
\hline $\begin{array}{l}\text { B. cenocepacia LMG } \\
16656 / J 2315\end{array}$ & $\begin{array}{l}\text { Human clinical specimen, } \\
\text { United Kingdom, } 1989\end{array}$ & [88] & $\begin{array}{l}\text { Uniprot database: } \\
\text { UP000001035 }\end{array}$ \\
\hline B. dolosa AU0158 & $\begin{array}{c}\text { Human clinical specimen, } \\
\text { USA unknown }\end{array}$ & [89] & $\begin{array}{l}\text { Uniprot database: } \\
\text { UP000032886 }\end{array}$ \\
\hline B. ubonensis MSMB22 & $\begin{array}{l}\text { Soil isolate, Australia, } \\
\qquad 2001\end{array}$ & [89] & $\begin{array}{c}\text { Burkholderia Genome } \\
\text { Database [90], } \\
\text { Strain number: } 3404\end{array}$ \\
\hline $\begin{array}{l}\text { C. fetus subsp. fetus NCTC } \\
10842\end{array}$ & $\begin{array}{c}\text { Brain of sheep fetus, } \\
\text { France, } 1952\end{array}$ & [91] & $\begin{array}{l}\text { Uniprot database: } \\
\text { UP000001035 }\end{array}$ \\
\hline
\end{tabular}


ASSOCIATED CONTENT

Supporting Information

Supplementary document: Containing Supplementary figures 1 to 5

Supplementary Figure 1: Identification of glycopeptides within B. cenocepacia J2315 whole proteome digests. A) Pie charts showing all unique PSMs identified with scores above 300 B) Number of glycopeptides PSMs (Byonic Score $>300$ ) identified within each unenriched sample.

Supplementary Figure 2: Manual assessment of glycopeptides identified with $B$. cenocepacia J2315 whole proteome digests. HCD and ETD fragmentation of novel glycoproteins A-B) B4E7L5; C-D) B4EBZ3; E-F) B4E732 and G-H) B4EB92.

Supplementary Figure 3: Analysis of B. cenocepacia glycopeptide identified within static FAIMS fractions. A) Scatter plot of CV vs $\mathrm{m} / \mathrm{z}$ of glycopeptide identified. B) Peptide properties of glycopeptide identified across static FAIMS CVs vs HILIC enriched glycopeptides

Supplementary Figure 4: Comparisons of glycopeptide length vs aliphaticity across the Burkholderia species $\boldsymbol{B}$. dolosa and B. ubonensis. Within strains examined difference in aliphaticity properties or length are observed from glycopeptides identified within stepped FAIMS and unenriched samples compared to ZIC-HILIC enrichments.

Supplementary Figure 5: Comparisons of glycopeptide length vs aliphaticity within $\boldsymbol{C}$. fetus subsp. fetus glycopeptide. No differences in the aliphatic properties or length are observed from glycopeptides identified within stepped FAIMS and unenriched samples compared to ZIC-HILIC enrichments.

Supplementary Tables: All Supplementary tables are provided within a single zip file

Supplementary table 1: Combined Byonic searches of B. cenocepacia J2315 whole proteome digests. The combined Byonic results of three technical replicates of B. cenocepacia J2315 showing all glycopeptides identified with a Byonic score over 300.

Supplementary table 2: Maxquant analysis of B. cenocepacia J2315. Maxquant analysis of three technical replicates of $B$. cenocepacia $\mathrm{J} 2315$ whole proteome digests.

Supplementary table 3: iBAQ measurements of B. cenocepacia J2315. iBAQ values for all glycoproteins identified within enriched and unenriched samples of $B$. cenocepacia $\mathrm{J} 2315$ whole proteome digests.

Supplementary table 4: Peptide properties of B. cenocepacia J2315 glycopeptides identified within and without ZIC-HILIC enrichments. For each glycopeptide identified within unenriched and ZIC-HILIC enriched samples the length and aliphatic index is provided. 
568

569

570

571

572

573

574

575

576

577

578

579

580

581

582

583

584

585

586

587

588

589

590

591

592

593

594

595

596

597

598

599

600

601

602

603

604

605

606

607

608

609

610

Supplementary table 5: Combined Byonic searches of B. cenocepacia J2315 without FAIMS. The combined Byonic results of three technical replicates of B. cenocepacia $J 2315$ showing all glycopeptides identified with a Byonic score over 300.

Supplementary table 6: Combined Byonic searches of B. cenocepacia J2315 with static FAIMS CVs. The combined Byonic results of each CV from -20 to -90 (three technical replicates of each) for B. cenocepacia J2315 showing all glycopeptides identified with a Byonic score over 300.

Supplementary table 7: Combined Byonic searches of B. cenocepacia J2315 with stepped FAIMS. The combined Byonic results for three technical replicates of B. cenocepacia J2315 with stepped FAIMS showing all glycopeptides identified with a Byonic score over 300.

Supplementary table 8: Peptide properties of glycopeptides identified within B. cenocepacia J2315 across analysis approaches. For each glycopeptide identified within stepped FAIMS, unenriched and ZIC-HILIC enriched samples the length and aliphatic index are provided.

Supplementary table 9: Combined Byonic searches of B. ubonensis MSMB22 with stepped FAIMS. The combined Byonic results for three technical replicates of $B$. ubonensis MSMB22 with stepped FAIMS showing all glycopeptides identified with a Byonic score over 300.

Supplementary table 10: Combined Byonic searches of B. ubonensis MSMB22 without FAIMS. The combined Byonic results of three technical replicates of $B$. ubonensis MSMB22 showing all glycopeptides identified with a Byonic score over 300.

Supplementary table 11: Combined Byonic searches of B. dolosa AU0158 with stepped FAIMS. The combined Byonic results for three technical replicates of B. dolosa AU0158with stepped FAIMS showing all glycopeptides identified with a Byonic score over 300.

Supplementary table 12: Combined Byonic searches of B. dolosa AU0158 without FAIMS. The combined Byonic results of three technical replicates of B. dolosa AU0158 showing all glycopeptides identified with a Byonic score over 300.

Supplementary table 13: Peptide properties of glycopeptides identified within $B$. ubonensis MSMB22 and B. dolosa AU0158 across analysis approaches. For each glycopeptide identified within stepped FAIMS, unenriched and ZIC-HILIC enriched samples the length and Aliphatic index is provided.

Supplementary table 14: Combined Byonic searches of C. fetus subsp. fetus NCTC 10842 with static FAIMS CVs. The combined Byonic results of each CV from -20 to -90 (three technical replicates) for C. fetus subsp. fetus NCTC 10842 showing all glycopeptides identified with a Byonic score over 300. 
611 Supplementary table 15: Combined Byonic searches of C. fetus subsp. fetus NCTC 10842

612 without FAIMS. The combined Byonic results of three technical replicates of $C$. fetus subsp. fetus

613 NCTC 10842 showing all glycopeptides identified with a Byonic score over 300.

614

615 Supplementary table 16: Combined Byonic searches of C. fetus subsp. fetus NCTC 10842 with

616 stepped FAIMS. The combined Byonic results for three technical replicates of $C$. fetus subsp. fetus 617 NCTC 10842 with stepped FAIMS showing all glycopeptides identified with a Byonic score over 618300.

Supplementary table 17: Peptide properties of glycopeptides identified within C. fetus subsp. fetus NCTC 10842 across analysis approaches. For each glycopeptide identified within stepped FAIMS, unenriched and ZIC-HILIC enriched samples the length and Aliphatic index is provided. 


\section{AUTHOR INFORMATION}

\section{Corresponding Author}

657 Dr Nichollas E. Scott

658 Email: Nichollas.scott@unimelb.edu.au

659 Address: Department of Microbiology and Immunology, University of Melbourne at the Peter

660 Doherty Institute for Infection and Immunity, Melbourne 3000, Australia

661

\section{Author contributions}

663 A.R.A.I prepared all sample for MS analysis. C.S.A, S.N and N.A.W maintained MS instrumentation

664 and provided access to critical resources. L.E.B assisted in manuscript preparation. N.E.S

665 conceived, designed all experiments, performed analysis, and undertook the preparation of the

666 manuscript.

667

668 Funding Sources

669 This work was supported by a National Health and Medical Research Council of Australia

670 (NHMRC) project grant awarded to NES (APP1100164).

671

\section{ACKNOWLEDGEMENTS}

673 We thank the Melbourne Mass Spectrometry and Proteomics Facility of The Bio21 Molecular

674 Science and Biotechnology Institute for access to MS instrumentation and Byonic. We would like

675 to thank Christine Szymanski and Justin Duma for the kind gift of Campylobacter fetus fetus NCTC 
67610842 lysates. We thank Ben Parker for feedback on the manuscript. We would also like to thank

677 Morten Thaysen-Andersen and Daniel Kolarich for their fruitful discussions and highlighting that

678 ZIC-HILIC enrichment was likely missing numerous Burkholderia glycopeptides. These

679 conversations were instrumental in leading to this work.

\section{ABBREVIATIONS}

682 Hydrophilic Interaction Liquid Chromatography (HILIC); Mass spectrometry (MS); Compensation

683 voltage (CV); Zwitterionic hydrophilic interaction liquid chromatography (ZIC-HILIC); galactose

684 (Gal); Glucose (Glc); N-Acetylgalactosamine (GaINAc); N-Acetylglucosamine (GlcNAc); Succinyl

685 (Suc); 2,4-diacetamido-2,4,6 trideoxyglucopyranose (diNAcBac); N-acetylhexoseamine (HexNAc);

686 Hexose (Hex); Ion Mobility Spectrometry (IMS); Field Asymmetric Waveform IMS (FAIMS); Luria

687 Bertani (LB); Phosphate-buffered saline (PBS); Sodium dodecyl sulfate (SDS); Normalized

688 collisional energy (NCE); Tris(hydroxymethyl)aminomethane (Tris); Trifluoroacetic acid (TFA);

689 Dithiothreitol (DTT); Higher-energy collision dissociation (HCD); Collision-induced dissociation

690 (CID); Electron-transfer/higher-energy collision dissociation (EThcD); Automatic Gain Control

691 (AGC); Dimethyl sulfoxide (DMSO); intensity-based absolute quantification (iBAQ); Peptide

692 spectrum matches (PSMs)

693

694

695

696

697

698

699

700

701 


\section{References}

703 1. Varki A, Cummings RD, Esko JD, Stanley P, Hart GW, Aebi M, et al., editors. Essentials of Glycobiology. 3rd ed: Cold Spring Harbor; 2015.

705 2. Yu A, Zhao J, Peng W, Banazadeh A, Williamson SD, Goli M, et al. Advances in mass 706 spectrometry-based glycoproteomics. Electrophoresis. 2018;39(24):3104-22. Epub 2018/09/12.

707 doi: 10.1002/elps.201800272. PubMed PMID: 30203847; PubMed Central PMCID: 708 PMCPMC6375712.

709 3. Xiao H, Sun F, Suttapitugsakul S, Wu R. Global and site-specific analysis of protein glycosylation in complex biological systems with Mass Spectrometry. Mass Spectrom Rev. 2019;38(4-5):356-79. Epub 2019/01/04. doi: 10.1002/mas.21586. PubMed PMID: 30605224; PubMed Central PMCID: PMCPMC6610820.

713 4. Thaysen-Andersen M, Packer NH, Schulz BL. Maturing Glycoproteomics Technologies

714 Provide Unique Structural Insights into the N-glycoproteome and Its Regulation in Health and

Disease. Molecular \& cellular proteomics : MCP. 2016;15(6):1773-90. doi: 10.1074/mcp.O115.057638. PubMed PMID: 26929216; PubMed Central PMCID: PMCPMC5083109.

718 5. Choi J, Wagner LJS, Timmermans S, Malaker SA, Schumann B, Gray MA, et al. Engineering Orthogonal Polypeptide GalNAc-Transferase and UDP-Sugar Pairs. J Am Chem Soc. 2019;141(34):13442-53. Epub 2019/08/03. doi: 10.1021/jacs.9b04695. PubMed PMID: 31373799; PubMed Central PMCID: PMCPMC6813768.

6. Schumann B, Malaker SA, Wisnovsky SP, Debets MF, Agbay AJ, Fernandez D, et al. Bump-and-Hole Engineering Identifies Specific Substrates of Glycosyltransferases in Living Cells. Mol Cell. 2020. Epub 2020/04/24. doi: 10.1016/j.molcel.2020.03.030. PubMed PMID: 32325029.

7. Steentoft C, Vakhrushev SY, Vester-Christensen MB, Schjoldager KT, Kong Y, Bennett $\mathrm{EP}$, et al. Mining the O-glycoproteome using zinc-finger nuclease-glycoengineered SimpleCell lines. Nature methods. 2011;8(11):977-82. Epub 2011/10/11. doi: 10.1038/nmeth.1731. PubMed PMID: 21983924.

8. Steentoft C, Vakhrushev SY, Joshi HJ, Kong Y, Vester-Christensen MB, Schjoldager KT, et al. Precision mapping of the human O-GalNAc glycoproteome through SimpleCell technology. EMBO J. 2013;32(10):1478-88. Epub 2013/04/16. doi: 10.1038/emboj.2013.79. PubMed PMID: 23584533; PubMed Central PMCID: PMCPMC3655468.

9. Vester-Christensen MB, Halim A, Joshi HJ, Steentoft C, Bennett EP, Levery SB, et al. Mining the O-mannose glycoproteome reveals cadherins as major O-mannosylated glycoproteins. Proceedings of the National Academy of Sciences of the United States of America. 2013;110(52):21018-23. Epub 2013/10/09. doi: 10.1073/pnas.1313446110. PubMed PMID: 24101494; PubMed Central PMCID: PMCPMC3876253.

10. Halim A, Larsen IS, Neubert P, Joshi HJ, Petersen BL, Vakhrushev SY, et al. Discovery of a nucleocytoplasmic O-mannose glycoproteome in yeast. Proceedings of the National Academy

741 of Sciences of the United States of America. 2015;112(51):15648-53. doi:

742 10.1073/pnas.1511743112. PubMed PMID: 26644575; PubMed Central PMCID:

743 PMCPMC4697373.

744 11. Woo CM, Lund PJ, Huang AC, Davis MM, Bertozzi CR, Pitteri SJ. Mapping and 745 Quantification of Over 2000 O-linked Glycopeptides in Activated Human T Cells with Isotope746 Targeted Glycoproteomics (Isotag). Molecular \& cellular proteomics : MCP. 2018;17(4):764-75. 
Epub 2018/01/21. doi: 10.1074/mcp.RA117.000261. PubMed PMID: 29351928; PubMed Central PMCID: PMCPMC5880114.

12. Wohlgemuth J, Karas M, Eichhorn T, Hendriks R, Andrecht S. Quantitative site-specific analysis of protein glycosylation by LC-MS using different glycopeptide-enrichment strategies. Anal Biochem. 2009;395(2):178-88. Epub 2009/08/25. doi: 10.1016/j.ab.2009.08.023. PubMed PMID: 19699707.

13. Hart-Smith G, Raftery MJ. Detection and characterization of low abundance glycopeptides via higher-energy C-trap dissociation and orbitrap mass analysis. J Am Soc Mass Spectrom. 2012;23(1):124-40. Epub 2011/11/16. doi: 10.1007/s13361-011-0273-y. PubMed PMID: 22083589.

14. Chen CC, Su WC, Huang BY, Chen YJ, Tai HC, Obena RP. Interaction modes and approaches to glycopeptide and glycoprotein enrichment. Analyst. 2014;139(4):688-704. Epub 2013/12/18. doi: 10.1039/c3an01813j. PubMed PMID: 24336240.

760 15. Zielinska DF, Gnad F, Schropp K, Wisniewski JR, Mann M. Mapping N-glycosylation sites across seven evolutionarily distant species reveals a divergent substrate proteome despite a common core machinery. Mol Cell. 2012;46(4):542-8. Epub 2012/05/29. doi: 10.1016/j.molcel.2012.04.031. PubMed PMID: 22633491.

764 16. Zielinska DF, Gnad F, Wisniewski JR, Mann M. Precision mapping of an in vivo Nglycoproteome reveals rigid topological and sequence constraints. Cell. 2010;141(5):897-907. Epub 2010/06/01. doi: 10.1016/j.cell.2010.04.012. PubMed PMID: 20510933.

17. Trinidad JC, Schoepfer R, Burlingame AL, Medzihradszky KF. N- and O-glycosylation in the murine synaptosome. Molecular \& cellular proteomics : MCP. 2013;12(12):3474-88. Epub 2013/07/03. doi: 10.1074/mcp.M113.030007. PubMed PMID: 23816992; PubMed Central PMCID: PMCPMC3861701.

18. Xiao H, Chen W, Smeekens JM, Wu R. An enrichment method based on synergistic and reversible covalent interactions for large-scale analysis of glycoproteins. Nat Commun. 2018;9(1):1692. Epub 2018/04/29. doi: 10.1038/s41467-018-04081-3. PubMed PMID: 29703890; PubMed Central PMCID: PMCPMC5923262.

19. Nilsson J, Ruetschi U, Halim A, Hesse C, Carlsohn E, Brinkmalm G, et al. Enrichment of glycopeptides for glycan structure and attachment site identification. Nature methods. 2009;6(11):809-11. Epub 2009/10/20. doi: 10.1038/nmeth.1392. PubMed PMID: 19838169. 20. Hagglund P, Bunkenborg J, Elortza F, Jensen ON, Roepstorff P. A new strategy for identification of $\mathrm{N}$-glycosylated proteins and unambiguous assignment of their glycosylation sites using HILIC enrichment and partial deglycosylation. Journal of proteome research. 2004;3(3):55666. Epub 2004/07/16. PubMed PMID: 15253437.

783

784

785

21. Jandera P. Stationary and mobile phases in hydrophilic interaction chromatography: a review. Anal Chim Acta. 2011;692(1-2):1-25. Epub 2011/04/20. doi: 10.1016/j.aca.2011.02.047. PubMed PMID: 21501708.

22. Jandera P, Janas P. Recent advances in stationary phases and understanding of retention in hydrophilic interaction chromatography. A review. Anal Chim Acta. 2017;967:12-32. Epub 2017/04/10. doi: 10.1016/j.aca.2017.01.060. PubMed PMID: 28390482.

790 23. Mysling S, Palmisano G, Hojrup P, Thaysen-Andersen M. Utilizing ion-pairing hydrophilic interaction chromatography solid phase extraction for efficient glycopeptide enrichment in glycoproteomics. Anal Chem. 2010;82(13):5598-609. Epub 2010/06/12. doi: 10.1021/ac100530w. PubMed PMID: 20536156. 
24. Hagglund P, Matthiesen R, Elortza F, Hojrup P, Roepstorff P, Jensen ON, et al. An enzymatic deglycosylation scheme enabling identification of core fucosylated N-glycans and Oglycosylation site mapping of human plasma proteins. Journal of proteome research. 2007;6(8):3021-31. Epub 2007/07/20. doi: 10.1021/pr0700605. PubMed PMID: 17636988. specific glycan-peptide analysis for determination of N-glycoproteome heterogeneity. Journal of proteome research. 2013;12(12):5791-800. Epub 2013/10/05. doi: 10.1021/pr400783j. PubMed PMID: 24090084.

26. Parker BL, Thaysen-Andersen M, Fazakerley DJ, Holliday M, Packer NH, James DE. Terminal Galactosylation and Sialylation Switching on Membrane Glycoproteins upon TNFAlpha-Induced Insulin Resistance in Adipocytes. Molecular \& cellular proteomics : MCP. 2016;15(1):141-53. Epub 2015/11/06. doi: 10.1074/mcp.M115.054221. PubMed PMID: 26537798 ; PubMed Central PMCID: PMCPMC4762517.

27. Loke I, Ostergaard O, Heegaard NHH, Packer NH, Thaysen-Andersen M. PaucimannoseRich N-glycosylation of Spatiotemporally Regulated Human Neutrophil Elastase Modulates Its Immune Functions. Molecular \& cellular proteomics : MCP. 2017;16(8):1507-27. Epub 2017/06/21. doi: 10.1074/mcp.M116.066746. PubMed PMID: 28630087; PubMed Central PMCID: PMCPMC5546201.

28. Lee LY, Moh ES, Parker BL, Bern M, Packer NH, Thaysen-Andersen M. Toward Automated N-Glycopeptide Identification in Glycoproteomics. Journal of proteome research. 2016;15(10):3904-15. Epub 2016/08/16. doi: 10.1021/acs.jproteome.6b00438. PubMed PMID: 27519006.

29. Scott NE, Parker BL, Connolly AM, Paulech J, Edwards AV, Crossett B, et al. Simultaneous glycan-peptide characterization using hydrophilic interaction chromatography and parallel fragmentation by CID, higher energy collisional dissociation, and electron transfer dissociation MS applied to the N-linked glycoproteome of Campylobacter jejuni. Molecular \& cellular proteomics : MCP. 2011;10(2):M000031-MCP201. doi: 10.1074/mcp.M000031MCP201. PubMed PMID: 20360033; PubMed Central PMCID: PMCPMC3033663.

30. Scott NE, Nothaft H, Edwards AV, Labbate M, Djordjevic SP, Larsen MR, et al. Modification of the Campylobacter jejuni N-linked glycan by EptC protein-mediated addition of phosphoethanolamine. The Journal of biological chemistry. 2012;287(35):29384-96. Epub 2012/07/05. doi: 10.1074/jbc.M112.380212. PubMed PMID: 22761430; PubMed Central PMCID: PMCPMC3436159.

31. Lithgow KV, Scott NE, Iwashkiw JA, Thomson EL, Foster LJ, Feldman MF, et al. A general protein O-glycosylation system within the Burkholderia cepacia complex is involved in motility and virulence. Molecular microbiology. 2014;92(1):116-37. doi: 10.1111/mmi.12540. PubMed PMID: 24673753.

32. Iwashkiw JA, Seper A, Weber BS, Scott NE, Vinogradov E, Stratilo C, et al. Identification of a general O-linked protein glycosylation system in Acinetobacter baumannii and its role in virulence and biofilm formation. PLoS pathogens. 2012;8(6):e1002758. doi: 10.1371/journal.ppat.1002758. PubMed PMID: 22685409; PubMed Central PMCID: PMC3369928.

836

33. Xue Y, Xie J, Fang P, Yao J, Yan G, Shen H, et al. Study on behaviors and performances of universal N-glycopeptide enrichment methods. Analyst. 2018;143(8):1870-80. Epub 2018/03/21. doi: 10.1039/C7AN02062G. PubMed PMID: 29557479. 
837 34. Neue K, Mormann M, Peter-Katalinic J, Pohlentz G. Elucidation of glycoprotein structures 838 by unspecific proteolysis and direct nanoESI mass spectrometric analysis of ZIC-HILIC-enriched glycopeptides. Journal of proteome research. 2011;10(5):2248-60. Epub 2011/03/30. doi: 10.1021/pr101082c. PubMed PMID: 21443200.

35. Fathy Mohamed Y, Scott NE, Molinaro A, Creuzenet C, Ortega X, Lertmemongkolchai G, et al. A general protein O-glycosylation machinery conserved in Burkholderia species improves bacterial fitness and elicits glycan immunogenicity in humans. The Journal of biological chemistry. 2019. Epub 2019/07/28. doi: 10.1074/jbc.RA119.009671. PubMed PMID: 31350337. 36. Nothaft H, Szymanski CM. New discoveries in bacterial N-glycosylation to expand the synthetic biology toolbox. Curr Opin Chem Biol. 2019;53:16-24. Epub 2019/07/10. doi: 10.1016/j.cbpa.2019.05.032. PubMed PMID: 31288139.

848 37. Szymanski CM, Wren BW. Protein glycosylation in bacterial mucosal pathogens. Nature reviews Microbiology. 2005;3(3):225-37. doi: 10.1038/nrmicro1100. PubMed PMID: 15738950. 38. Koomey M. O-linked protein glycosylation in bacteria: snapshots and current perspectives. Curr Opin Struct Biol. 2019;56:198-203. Epub 2019/05/13. doi: 10.1016/j.sbi.2019.03.020. PubMed PMID: 31078896.

39. Joshi HJ, Narimatsu Y, Schjoldager KT, Tytgat HLP, Aebi M, Clausen H, et al. SnapShot: O-Glycosylation Pathways across Kingdoms. Cell. 2018;172(3):632- e2. Epub 2018/01/27. doi: 10.1016/j.cell.2018.01.016. PubMed PMID: 29373833.

856 40. Elhenawy W, Scott NE, Tondo ML, Orellano EG, Foster LJ, Feldman MF. Protein Olinked glycosylation in the plant pathogen Ralstonia solanacearum. Glycobiology. 2016;26(3):301-11. doi: 10.1093/glycob/cwv098. PubMed PMID: 26531228; PubMed Central PMCID: PMCPMC4736539.

860 41. Harding CM, Nasr MA, Kinsella RL, Scott NE, Foster LJ, Weber BS, et al. Acinetobacter strains carry two functional oligosaccharyltransferases, one devoted exclusively to type IV pilin, and the other one dedicated to O-glycosylation of multiple proteins. Molecular microbiology. 2015;96(5):1023-41. Epub 2015/03/03. doi: 10.1111/mmi.12986. PubMed PMID: 25727908. the O-linked protein glycosylation systems of acinetobacter species. Molecular \& cellular proteomics : MCP. 2014;13(9):2354-70. doi: 10.1074/mcp.M114.038315. PubMed PMID: 24917611 ; PubMed Central PMCID: PMC4159654.

868 43. Scott NE, Marzook NB, Cain JA, Solis N, Thaysen-Andersen M, Djordjevic SP, et al. Comparative proteomics and glycoproteomics reveal increased $\mathrm{N}$-linked glycosylation and relaxed sequon specificity in Campylobacter jejuni NCTC11168 O. Journal of proteome research. 2014;13(11):5136-50. Epub 2014/08/06. doi: 10.1021/pr5005554. PubMed PMID: 25093254.

873

874 44. Ahmad Izaham AR, Scott NE. Open database searching enables the identification and comparison of bacterial glycoproteomes without defining glycan compositions prior to searching. Molecular \& cellular proteomics : MCP. 2020. Epub 2020/06/25. doi: 10.1074/mcp.TIR120.002100. PubMed PMID: 32576591.

876 45. Oppy CC, Jebeli L, Kuba M, Oates CV, Strugnell R, Edgington-Mitchell LE, et al. Loss of O-linked protein glycosylation in Burkholderia cenocepacia impairs biofilm formation, siderophore activity and alters transcriptional regulators mSphere. 2019;(Accepted).

880 emerging pathogen, Burkholderia pseudomallei, depends upon the O-linked 881 oligosaccharyltransferase, PglL. Future Microbiol. 2020;15:241-57. Epub 2020/04/10. doi: 
883

884

885

886

887

888

889

890

891

892

893

894

895

896

897

898

899

900

901

902

903

904

905

906

907

908

909

910

911

912

913

914

915

916

917

918

919

920

921

922

923

924

925

926

47. Cumeras R, Figueras E, Davis CE, Baumbach JI, Gracia I. Review on ion mobility spectrometry. Part 1: current instrumentation. Analyst. 2015;140(5):1376-90. Epub 2014/12/04. doi: 10.1039/c4an01100g. PubMed PMID: 25465076; PubMed Central PMCID: PMCPMC4331213.

48. Winter DL, Wilkins MR, Donald WA. Differential Ion Mobility-Mass Spectrometry for Detailed Analysis of the Proteome. Trends Biotechnol. 2019;37(2):198-213. Epub 2018/09/09. doi: 10.1016/j.tibtech.2018.07.018. PubMed PMID: 30193737.

49. Pfammatter S, Bonneil E, McManus FP, Prasad S, Bailey DJ, Belford M, et al. A Novel Differential Ion Mobility Device Expands the Depth of Proteome Coverage and the Sensitivity of Multiplex Proteomic Measurements. Molecular \& cellular proteomics : MCP. 2018;17(10):205167. Epub 2018/07/17. doi: 10.1074/mcp.TIR118.000862. PubMed PMID: 30007914; PubMed Central PMCID: PMCPMC6166672.

50. Hebert AS, Prasad S, Belford MW, Bailey DJ, McAlister GC, Abbatiello SE, et al. Comprehensive Single-Shot Proteomics with FAIMS on a Hybrid Orbitrap Mass Spectrometer. Anal Chem. 2018;90(15):9529-37. Epub 2018/07/04. doi: 10.1021/acs.analchem.8b02233. PubMed PMID: 29969236; PubMed Central PMCID: PMCPMC6145172.

51. Bekker-Jensen DB, Martinez-Val A, Steigerwald S, Ruther P, Fort KL, Arrey TN, et al. A Compact Quadrupole-Orbitrap Mass Spectrometer with FAIMS Interface Improves Proteome Coverage in Short LC Gradients. Molecular \& cellular proteomics : MCP. 2020;19(4):716-29. Epub 2020/02/14. doi: 10.1074/mcp.TIR119.001906. PubMed PMID: 32051234; PubMed Central PMCID: PMCPMC7124470.

52. Schweppe DK, Prasad S, Belford MW, Navarrete-Perea J, Bailey DJ, Huguet R, et al. Characterization and Optimization of Multiplexed Quantitative Analyses Using High-Field Asymmetric-Waveform Ion Mobility Mass Spectrometry. Anal Chem. 2019;91(6):4010-6. Epub 2019/01/24. doi: 10.1021/acs.analchem.8b05399. PubMed PMID: 30672687; PubMed Central PMCID: PMCPMC6993951.

53. Bridon G, Bonneil E, Muratore-Schroeder T, Caron-Lizotte O, Thibault P. Improvement of phosphoproteome analyses using FAIMS and decision tree fragmentation. application to the insulin signaling pathway in Drosophila melanogaster S2 cells. Journal of proteome research. 2012;11(2):927-40. Epub 2011/11/09. doi: 10.1021/pr200722s. PubMed PMID: 22059388.

54. Shvartsburg AA, Zheng Y, Smith RD, Kelleher NL. Separation of variant methylated histone tails by differential ion mobility. Anal Chem. 2012;84(15):6317-20. Epub 2012/07/21. doi: 10.1021/ac301541r. PubMed PMID: 22812477; PubMed Central PMCID: PMCPMC3418378.

55. Pfammatter S, Bonneil E, McManus FP, Thibault P. Gas-Phase Enrichment of Multiply Charged Peptide Ions by Differential Ion Mobility Extend the Comprehensiveness of SUMO Proteome Analyses. J Am Soc Mass Spectrom. 2018;29(6):1111-24. Epub 2018/04/07. doi: 10.1007/s13361-018-1917-y. PubMed PMID: 29623662.

56. Schnirch L, Nadler-Holly M, Siao SW, Frese CK, Viner R, Liu F. Expanding the depth and sensitivity of cross-link identification by differential ion mobility using FAIMS. Anal Chem. 2020. Epub 2020/07/10. doi: 10.1021/acs.analchem.0c01273. PubMed PMID: 32643919.

57. Creese AJ, Cooper HJ. Separation and identification of isomeric glycopeptides by high field asymmetric waveform ion mobility spectrometry. Anal Chem. 2012;84(5):2597-601. Epub 2012/01/28. doi: 10.1021/ac203321y. PubMed PMID: 22280549; PubMed Central PMCID: PMCPMC3295202. 
58. Pathak P, Baird MA, Shvartsburg AA. High-Resolution Ion Mobility Separations of Isomeric Glycoforms with Variations on the Peptide and Glycan Levels. J Am Soc Mass Spectrom. 2020. Epub 2020/06/06. doi: 10.1021/jasms.0c00183. PubMed PMID: 32501708.

59. Ulasi GN, Creese AJ, Hui SX, Penn CW, Cooper HJ. Comprehensive mapping of Oglycosylation in flagellin from Campylobacter jejuni 11168: A multienzyme differential ion mobility mass spectrometry approach. Proteomics. 2015;15(16):2733-45. Epub 2015/04/18. doi: 10.1002/pmic.201400533. PubMed PMID: 25884275; PubMed Central PMCID: PMCPMC4975691.

60. Gelb AS, Lai R, Li H, Dodds ED. Composition and charge state influence on the ionneutral collision cross sections of protonated N-linked glycopeptides: an experimental and theoretical deconstruction of coulombic repulsion vs. charge solvation effects. Analyst. 2019;144(19):5738-47. Epub 2019/08/28. doi: 10.1039/c9an00875f. PubMed PMID: 31453603; PubMed Central PMCID: PMCPMC6756964.

61. Glaskin RS, Khatri K, Wang Q, Zaia J, Costello CE. Construction of a Database of Collision Cross Section Values for Glycopeptides, Glycans, and Peptides Determined by IM-MS. Anal Chem. 2017;89(8):4452-60. Epub 2017/03/23. doi: 10.1021/acs.analchem.6b04146. PubMed PMID: 28323417; PubMed Central PMCID: PMCPMC5766263.

62. Li H, Bendiak B, Siems WF, Gang DR, Hill HH, Jr. Ion Mobility-Mass Correlation Trend Line Separation of Glycoprotein Digests without Deglycosylation. Int J Ion Mobil Spectrom. 2013;16(2):105-15. Epub 2013/08/06. doi: 10.1007/s12127-013-0127-3. PubMed PMID: 23914139; PubMed Central PMCID: PMCPMC3728182.

63. Bzdyl NM, Scott NE, Norville IH, Scott AE, Atkins T, Pang S, et al. Peptidyl-Prolyl Isomerase ppiB Is Essential for Proteome Homeostasis and Virulence in Burkholderia pseudomallei. Infection and immunity. 2019;87(10). Epub 2019/07/25. doi: 10.1128/IAI.0052819. PubMed PMID: 31331957; PubMed Central PMCID: PMCPMC6759293.

64. Nothaft H, Scott NE, Vinogradov E, Liu X, Hu R, Beadle B, et al. Diversity in the protein $\mathrm{N}$-glycosylation pathways within the Campylobacter genus. Molecular \& cellular proteomics : MCP. 2012;11(11):1203-19. doi: 10.1074/mcp.M112.021519. PubMed PMID: 22859570; PubMed Central PMCID: PMC3494190.

65. Saba J, Dutta S, Hemenway E, Viner R. Increasing the productivity of glycopeptides analysis by using higher-energy collision dissociation-accurate mass-product-dependent electron transfer dissociation. Int J Proteomics. 2012;2012:560391. doi: 10.1155/2012/560391. PubMed PMID: 22701174; PubMed Central PMCID: PMCPMC3369405.

66. Bern M, Kil YJ, Becker C. Byonic: advanced peptide and protein identification software. Curr Protoc Bioinformatics. 2012; Chapter 13:Unit13 20. Epub 2012/12/21. doi: 10.1002/0471250953.bi1320s40. PubMed PMID: 23255153; PubMed Central PMCID: PMCPMC3545648.

67. Lenco J, Khalikova MA, Svec F. Dissolving Peptides in 0.1\% Formic Acid Brings Risk of Artificial Formylation. Journal of proteome research. 2020;19(3):993-9. Epub 2020/02/19. doi: 10.1021/acs.jproteome.9b00823. PubMed PMID: 32068400.

68. Cox J, Mann M. MaxQuant enables high peptide identification rates, individualized p.p.b.range mass accuracies and proteome-wide protein quantification. Nat Biotechnol. 2008;26(12):1367-72. doi: 10.1038/nbt.1511. PubMed PMID: 19029910.

69. Tyanova S, Temu T, Sinitcyn P, Carlson A, Hein MY, Geiger T, et al. The Perseus computational platform for comprehensive analysis of (prote)omics data. Nature methods. 2016;13(9):731-40. Epub 2016/06/28. doi: 10.1038/nmeth.3901. PubMed PMID: 27348712. 
973 70. Osorio D, Rondón-Villarreal P, Torres R. Peptides: A Package for Data Mining of Antimicrobial Peptides. The R Journal. 2015;7(1):10.

975 71. Wickham H. ggplot2: Elegant Graphics for Data Analysis: Springer-Verlag New York; 2016.

72. Ikai A. Thermostability and aliphatic index of globular proteins. J Biochem. 1980;88(6):1895-8. Epub 1980/12/01. PubMed PMID: 7462208.

979 73. Perez-Riverol Y, Csordas A, Bai J, Bernal-Llinares M, Hewapathirana S, Kundu DJ, et al. 980 The PRIDE database and related tools and resources in 2019: improving support for quantification data. Nucleic Acids Res. 2019;47(D1):D442-D50. Epub 2018/11/06. doi: 10.1093/nar/gky1106. PubMed PMID: 30395289; PubMed Central PMCID: PMCPMC6323896.

983 74. Vizcaino JA, Csordas A, del-Toro N, Dianes JA, Griss J, Lavidas I, et al. 2016 update of 984 the PRIDE database and its related tools. Nucleic Acids Res. 2016;44(D1):D447-56. doi: 10.1093/nar/gkv1145. PubMed PMID: 26527722; PubMed Central PMCID: PMCPMC4702828. 75. Alagesan K, Khilji SK, Kolarich D. It is all about the solvent: on the importance of the mobile phase for ZIC-HILIC glycopeptide enrichment. Anal Bioanal Chem. 2017;409(2):529-38. Epub 2016/12/03. doi: 10.1007/s00216-016-0051-6. PubMed PMID: 27909778; PubMed Central PMCID: PMCPMC5203826.

990 76. Hadjineophytou C, Anonsen JH, Wang N, Ma KC, Viburiene R, Vik A, et al. Genetic 991 determinants of genus-Level glycan diversity in a bacterial protein glycosylation system. PLoS 992 Genet. 2019;15(12):e1008532. Epub 2019/12/24. doi: 10.1371/journal.pgen.1008532. PubMed 993 PMID: 31869330.

994 77. Hahne H, Gholami AM, Kuster B. Discovery of O-GlcNAc-modified proteins in published 995 large-scale proteome data. Molecular \& cellular proteomics : MCP. 2012;11(10):843-50. Epub 996 2012/06/05. doi: 10.1074/mcp.M112.019463. PubMed PMID: 22661428; PubMed Central 997 PMCID: PMCPMC3494142.

998 78. Hahne H, Kuster B. Discovery of O-GlcNAc-6-phosphate modified proteins in large-scale phosphoproteomics data. Molecular \& cellular proteomics : MCP. 2012;11(10):1063-9. Epub

1001 2012/07/25. doi: 10.1074/mcp.M112.019760. PubMed PMID: 22826440; PubMed Central PMCID: PMCPMC3494138.

1002

1003

79. Chick JM, Kolippakkam D, Nusinow DP, Zhai B, Rad R, Huttlin EL, et al. A mass-tolerant

1004

1005 database search identifies a large proportion of unassigned spectra in shotgun proteomics as modified peptides. Nat Biotechnol. 2015;33(7):743-9. doi: 10.1038/nbt.3267. PubMed PMID: 26076430; PubMed Central PMCID: PMCPMC4515955.

1006 80. Li Q, Shortreed MR, Wenger CD, Frey BL, Schaffer LV, Scalf M, et al. Global PostTranslational Modification Discovery. Journal of proteome research. 2017;16(4):1383-90. Epub 2017/03/02. doi: 10.1021/acs.jproteome.6b00034. PubMed PMID: 28248113; PubMed Central PMCID: PMCPMC5387672.

1010 81. Solntsev SK, Shortreed MR, Frey BL, Smith LM. Enhanced Global Post-translational Modification Discovery with MetaMorpheus. Journal of proteome research. 2018;17(5):1844-51. Epub 2018/03/27. doi: 10.1021/acs.jproteome.7b00873. PubMed PMID: 29578715.

1013 82. Stavenhagen K, Hinneburg H, Thaysen-Andersen M, Hartmann L, Varon Silva D, Fuchser $1014 \mathrm{~J}$, et al. Quantitative mapping of glycoprotein micro-heterogeneity and macro-heterogeneity: an evaluation of mass spectrometry signal strengths using synthetic peptides and glycopeptides. J 23722953. 
83. Schulz BL, Jen FE, Power PM, Jones CE, Fox KL, Ku SC, et al. Identification of bacterial protein O-oligosaccharyltransferases and their glycoprotein substrates. PloS one. 2013;8(5):e62768. Epub 2013/05/10. doi: 10.1371/journal.pone.0062768. PubMed PMID: 23658772; PubMed Central PMCID: PMC3643930.

1022

84. Gebhart C, Ielmini MV, Reiz B, Price NL, Aas FE, Koomey M, et al. Characterization of exogenous bacterial oligosaccharyltransferases in Escherichia coli reveals the potential for Olinked protein glycosylation in Vibrio cholerae and Burkholderia thailandensis. Glycobiology. 2012;22(7):962-74. doi: 10.1093/glycob/cws059. PubMed PMID: 22391990.

85. Vorkapic D, Mitterer F, Pressler K, Leitner DR, Anonsen JH, Liesinger L, et al. A Broad Spectrum Protein Glycosylation System Influences Type II Protein Secretion and Associated Phenotypes in Vibrio cholerae. Front Microbiol. 2019;10:2780. Epub 2019/12/19. doi: 10.3389/fmicb.2019.02780. PubMed PMID: 31849912; PubMed Central PMCID: PMCPMC6901666.

86. Jervis AJ, Wood AG, Cain JA, Butler JA, Frost H, Lord E, et al. Functional analysis of the

1032 Helicobacter pullorum N-linked protein glycosylation system. Glycobiology. 2018;28(4):233-44. Epub 2018/01/18. doi: 10.1093/glycob/cwx110. PubMed PMID: 29340583; PubMed Central PMCID: PMCPMC6025236.

1035

87. Jervis AJ, Butler JA, Lawson AJ, Langdon R, Wren BW, Linton D. Characterization of the structurally diverse N-linked glycans of Campylobacter species. Journal of bacteriology. 2012;194(9):2355-62. Epub 2012/03/06. doi: 10.1128/JB.00042-12. PubMed PMID: 22389484; PubMed Central PMCID: PMCPMC3347071.

1039 88. Vandamme P, Holmes B, Vancanneyt M, Coenye T, Hoste B, Coopman R, et al. Occurrence of multiple genomovars of Burkholderia cepacia in cystic fibrosis patients and proposal of Burkholderia multivorans sp. nov. Int J Syst Bacteriol. 1997;47(4):1188-200. Epub 1997/10/23. doi: 10.1099/00207713-47-4-1188. PubMed PMID: 9336927. Complete genome sequences for 59 burkholderia isolates, both pathogenic and near neighbor. Genome Announc. 2015;3(2). Epub 2015/05/02. doi: 10.1128/genomeA.00159-15. PubMed PMID: 25931592; PubMed Central PMCID: PMCPMC4417688. Burkholderia Genome Database: facilitating flexible queries and comparative analyses. Bioinformatics. 2008;24(23):2803-4. Epub 2008/10/10. doi: 10.1093/bioinformatics/btn524. PubMed PMID: 18842600; PubMed Central PMCID: PMCPMC2639269. 
A) Charge states of unenriched glycopeptides

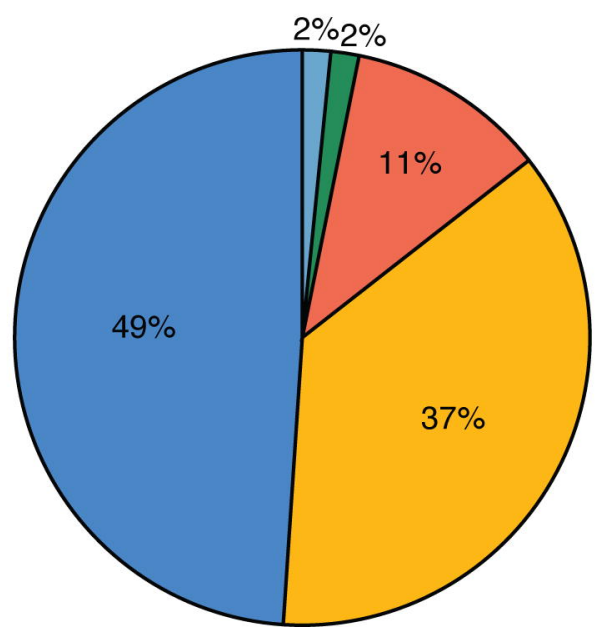

C) Unique Glycopeptides vs CV

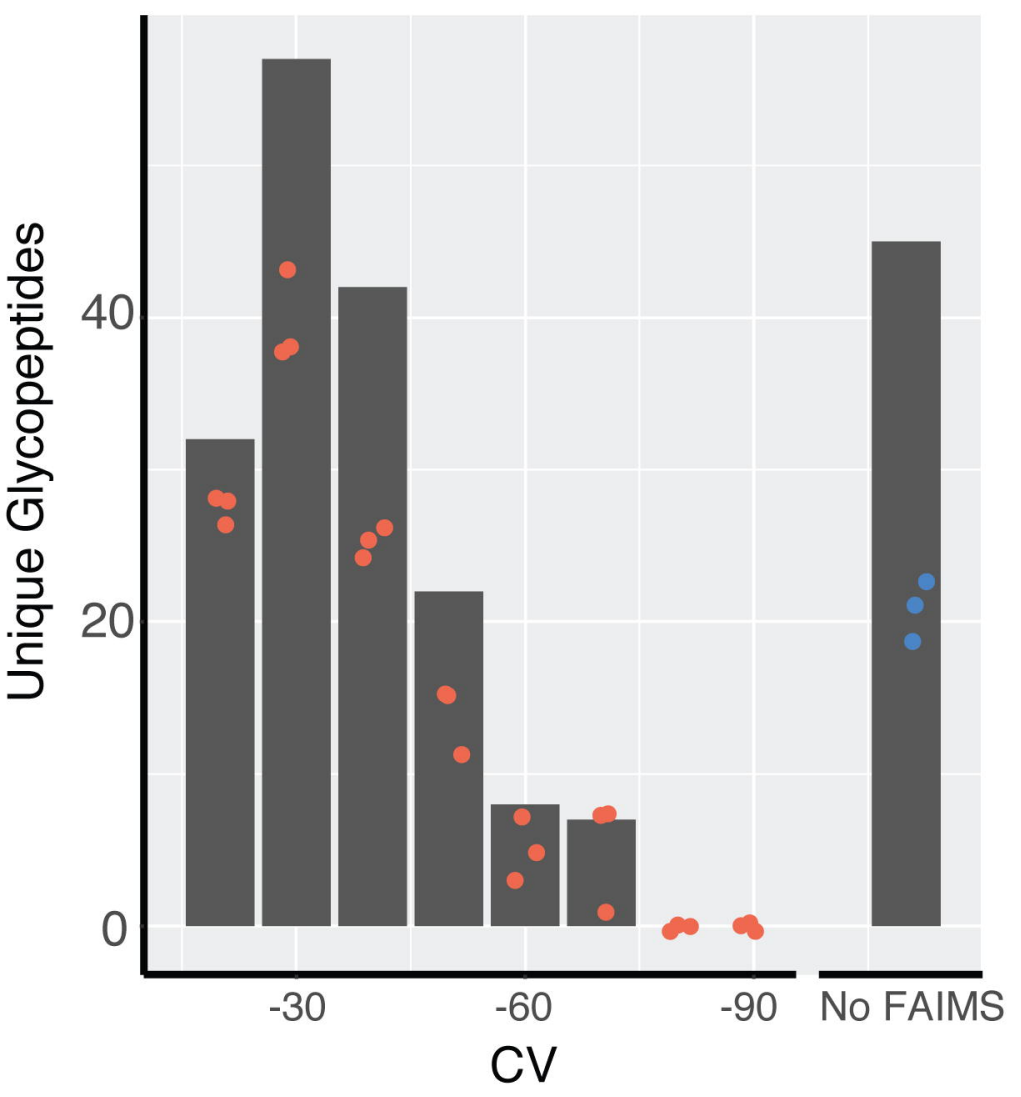

B) Charge states of enriched glycopeptides

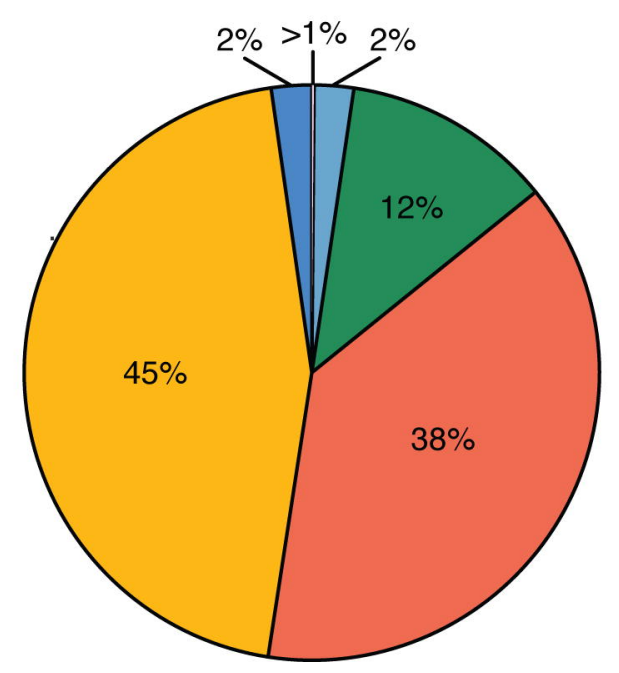

D) Glycoproteins

E)

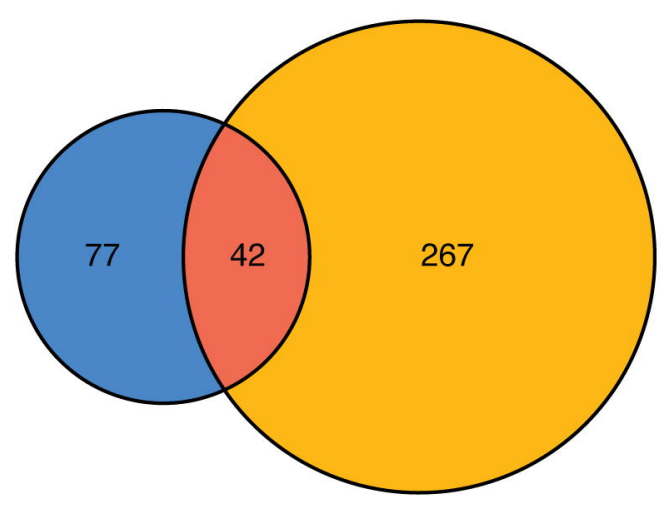

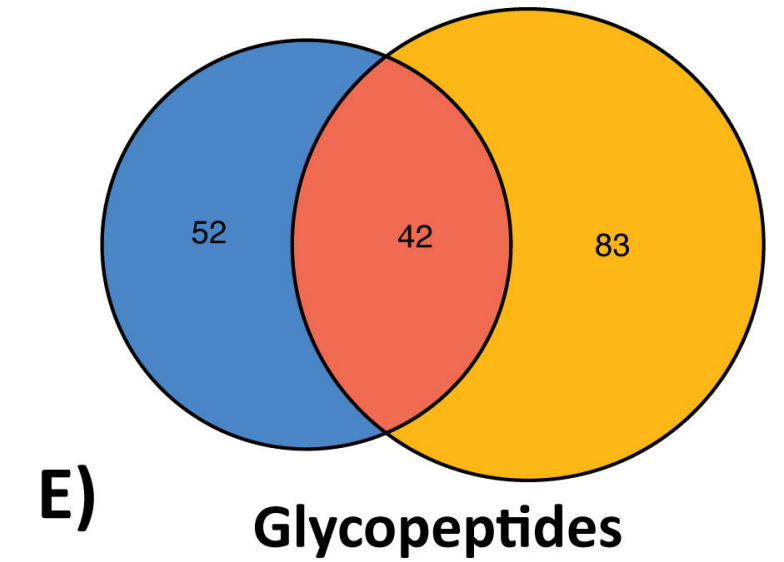

Charge

States

2

3

4

5

6

7

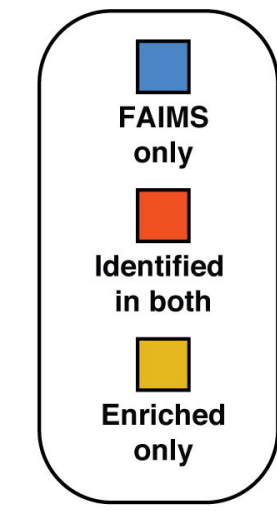


B) Unique glycopeptides observed
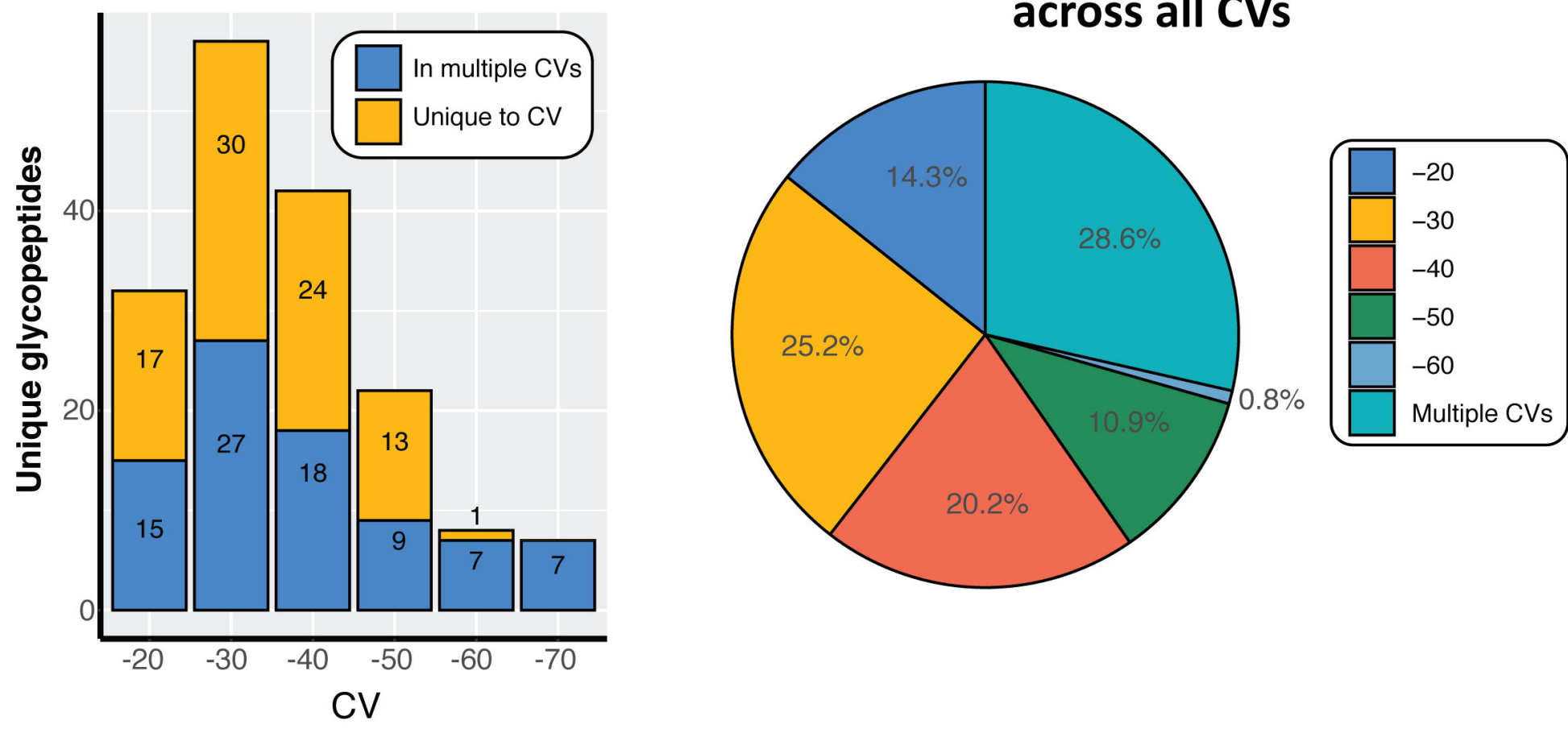


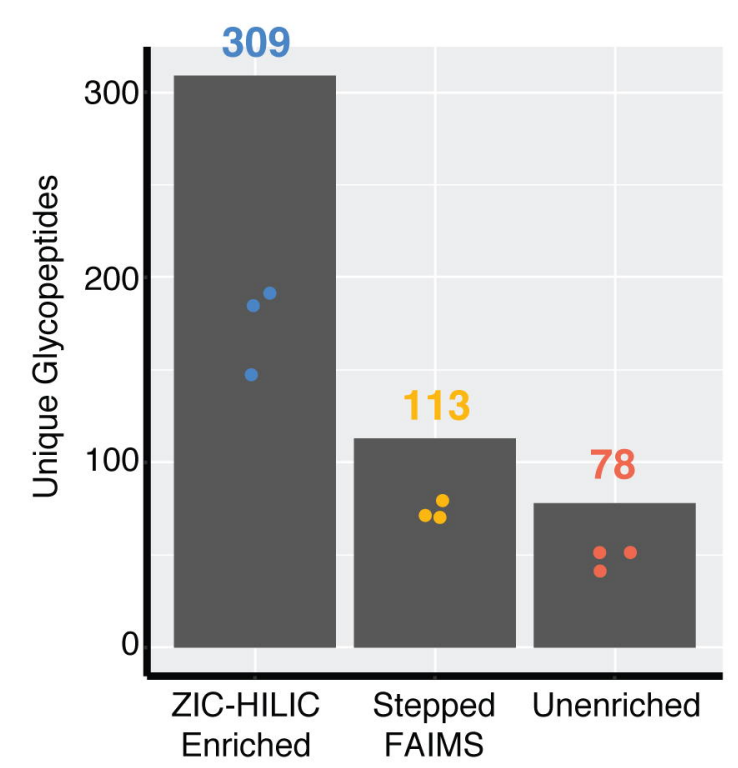

C) Glycopeptides Overlap

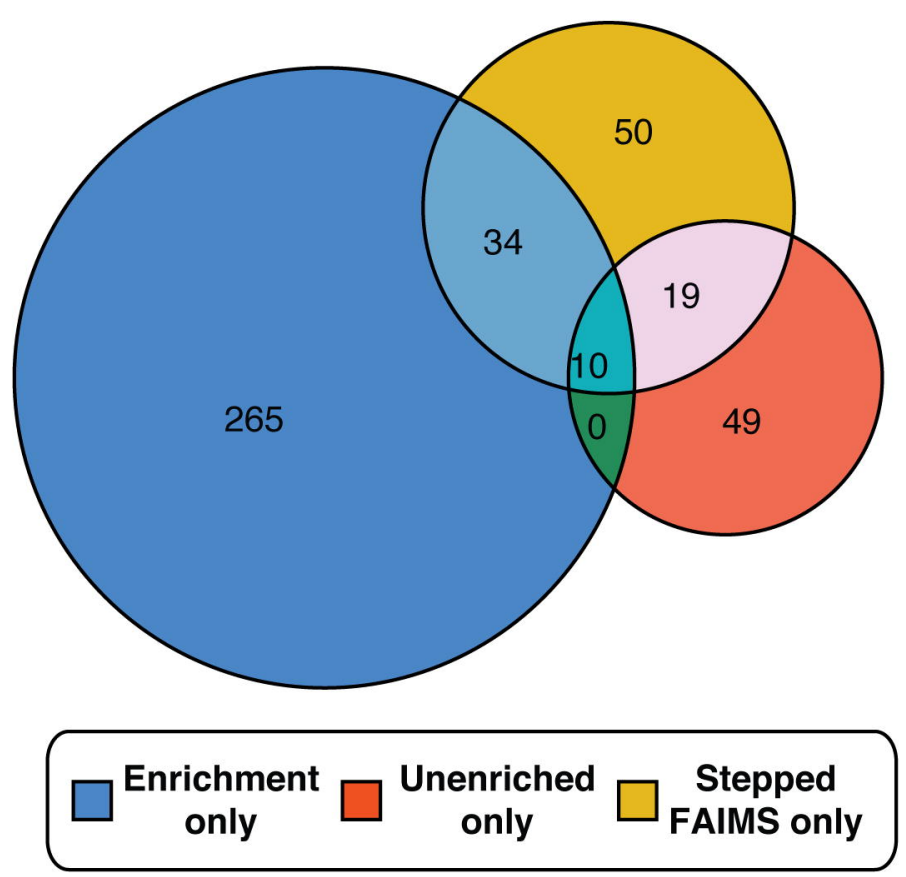

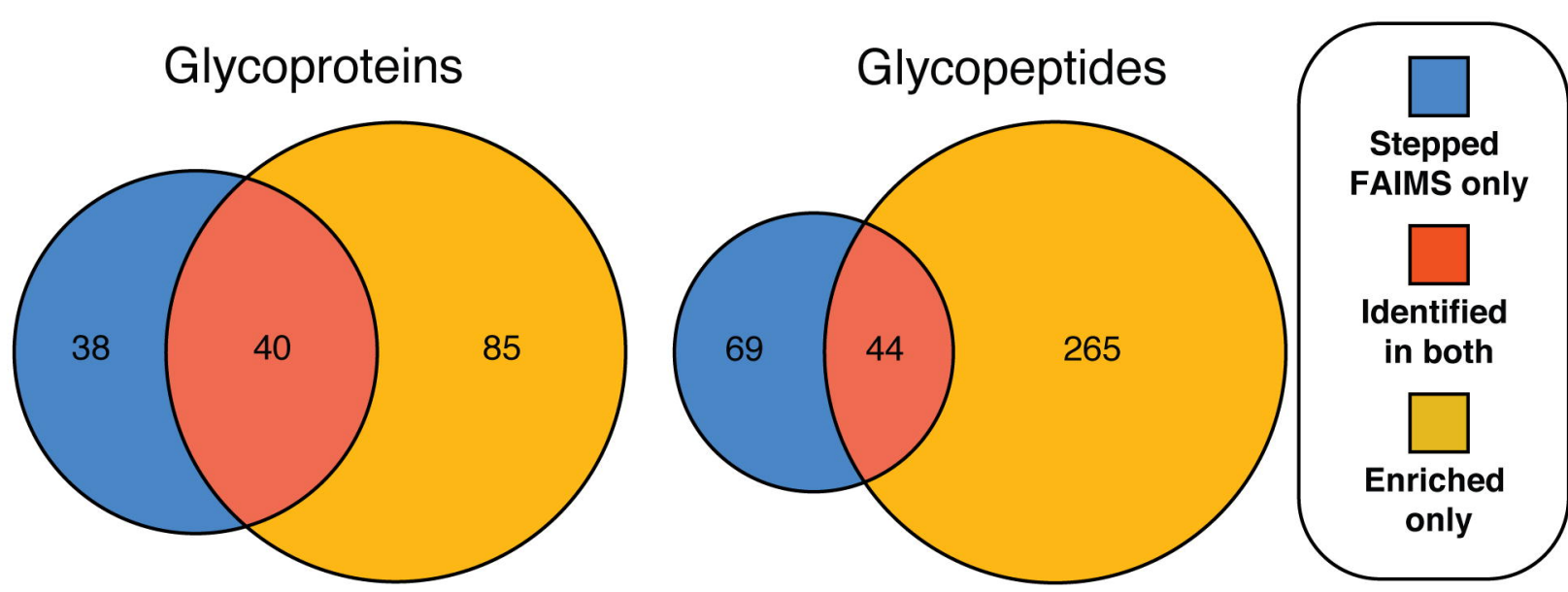

D)

\section{Peptide Properties}
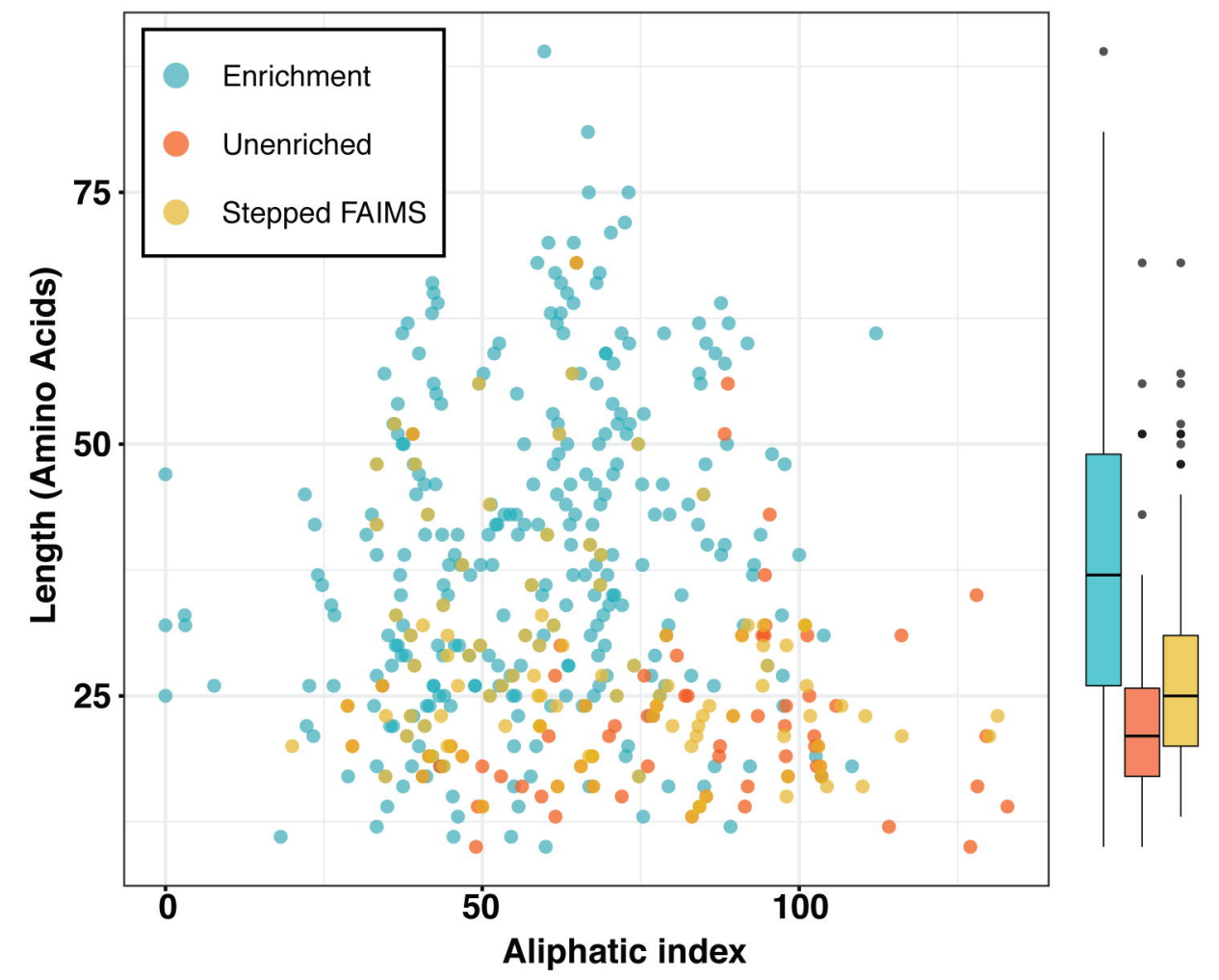
A) B. dolosa AU0158

\section{Glycoproteome}

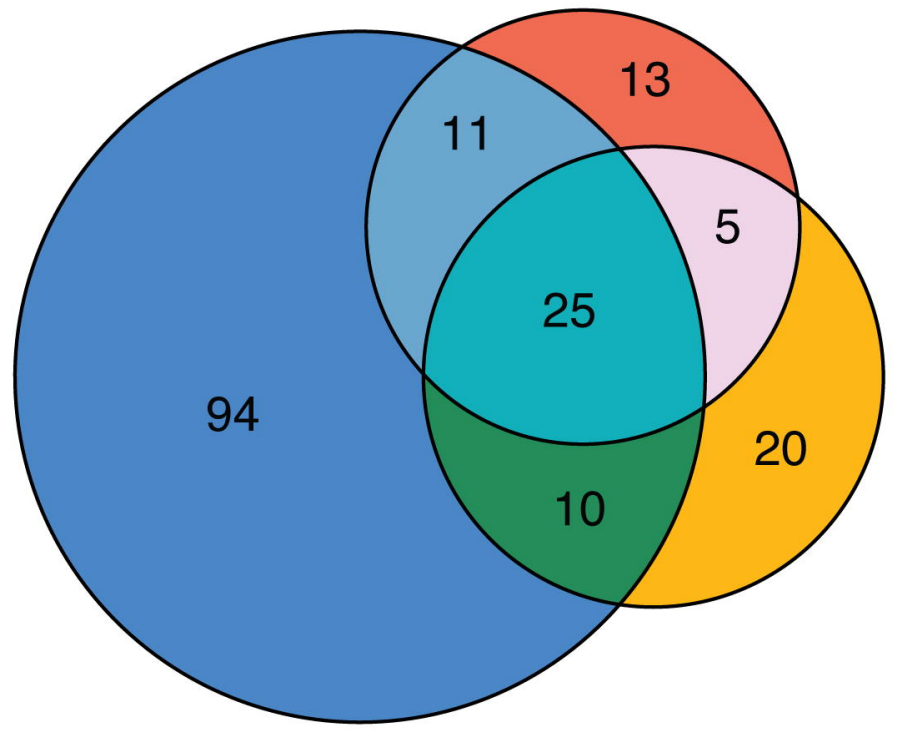

Glycopeptides

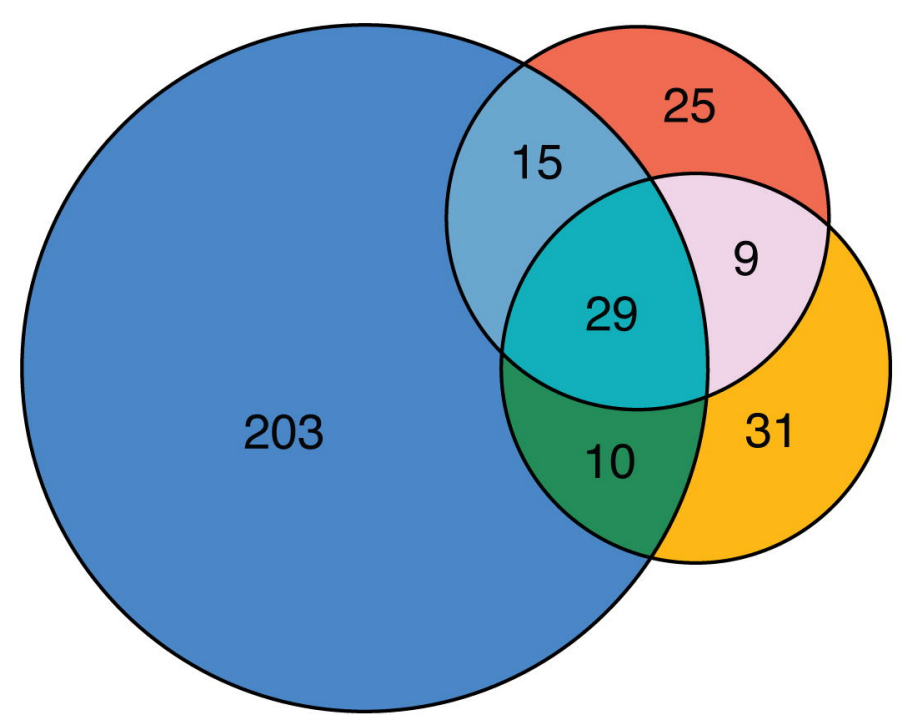

B) B. ubonensis MSMB22

\section{Glycoproteome}

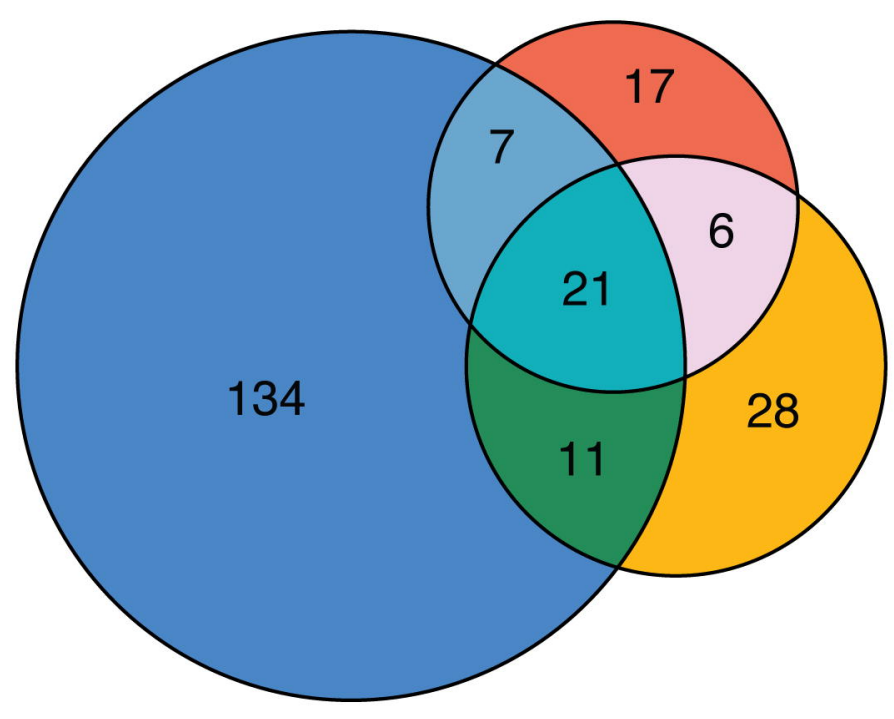

Glycopeptides

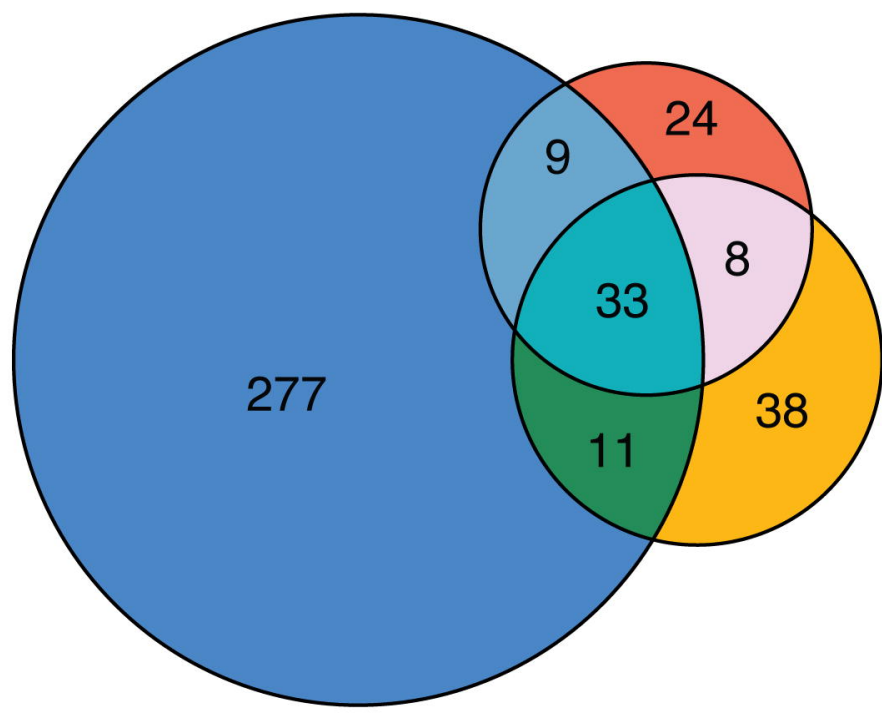

$\square$ Enrichment $\square$ Stepped $\square$ Unenriched only $\square$ FAIMS only $\square$ only 
A) Unique Glycopeptides vs CV

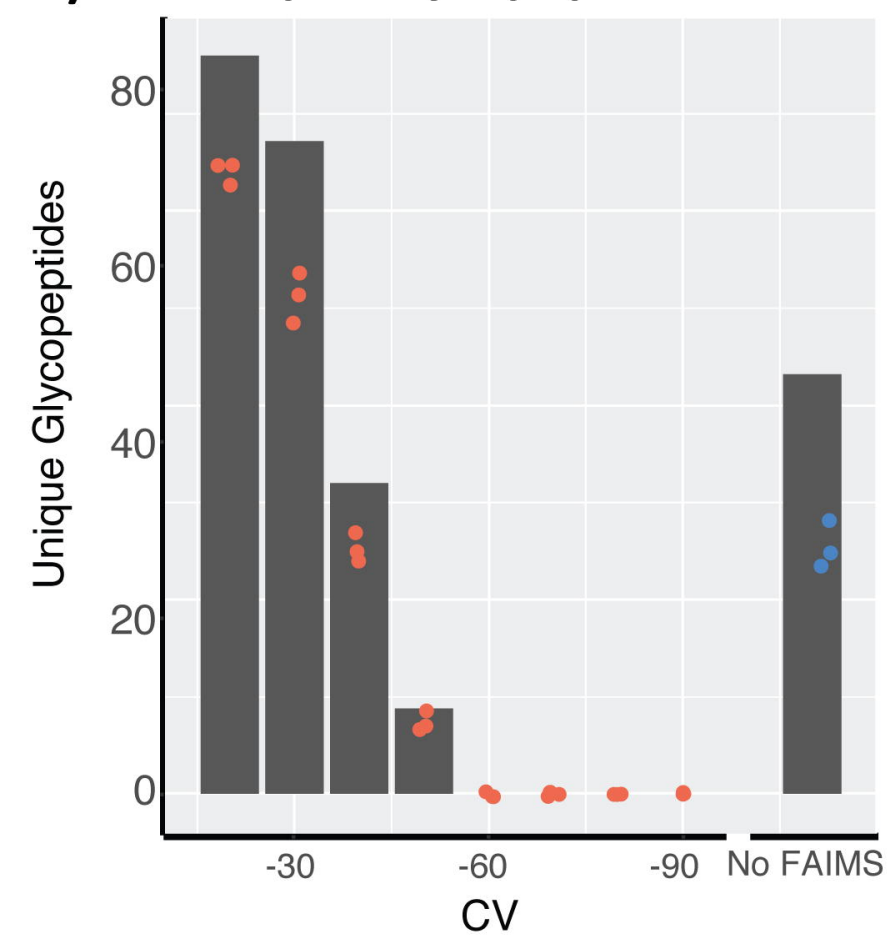

B)

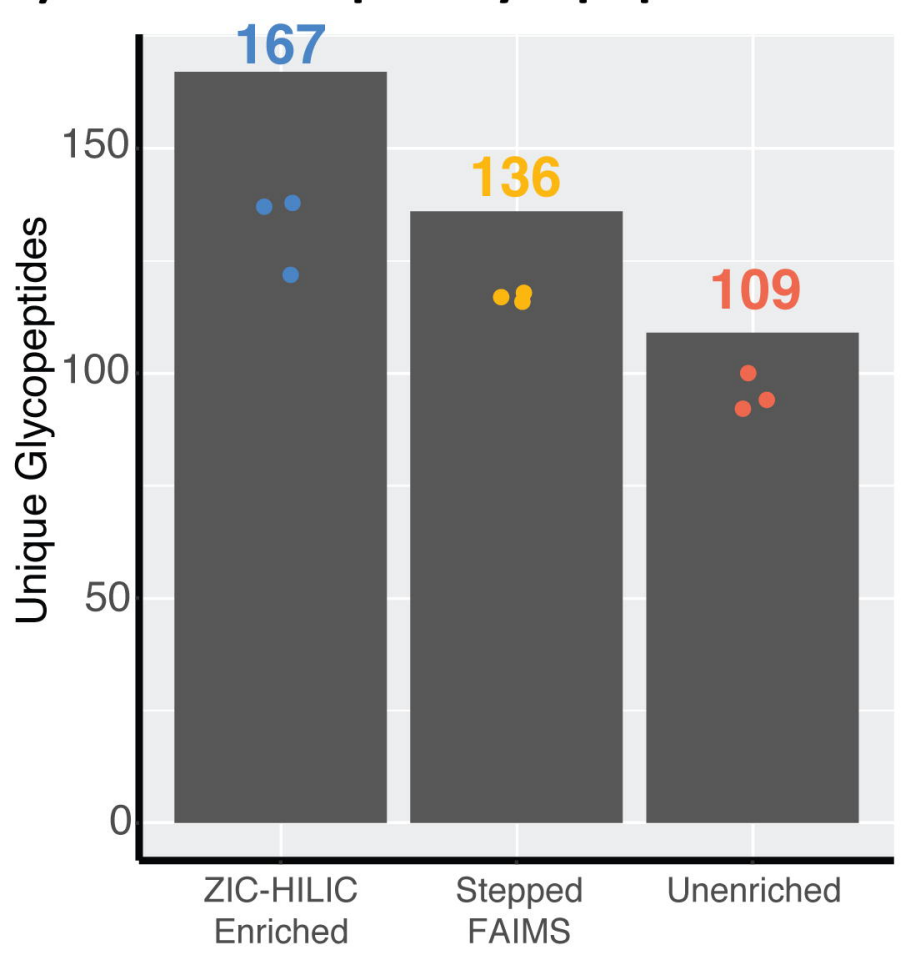

C) Stepped FAIMS vs ZIC-HILIC

\section{Glycopeptides}

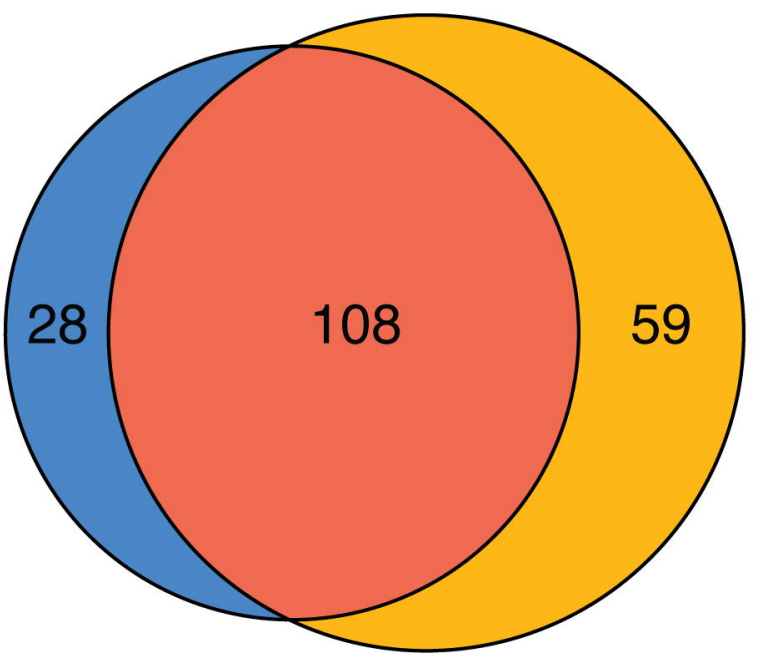

Glycoproteins
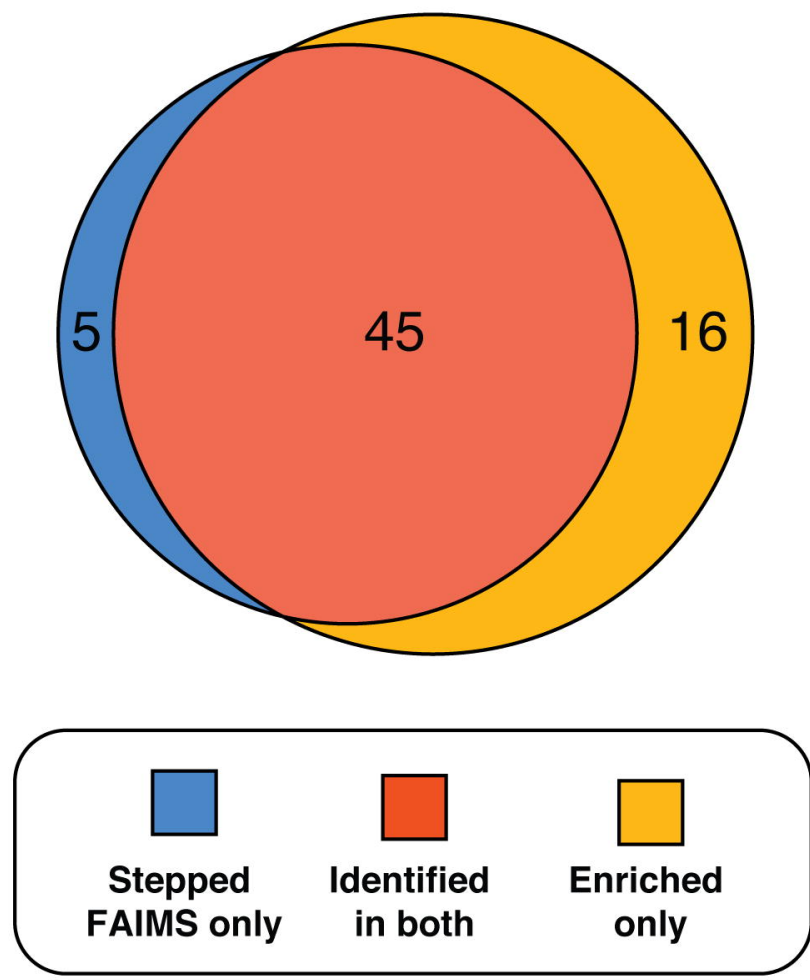